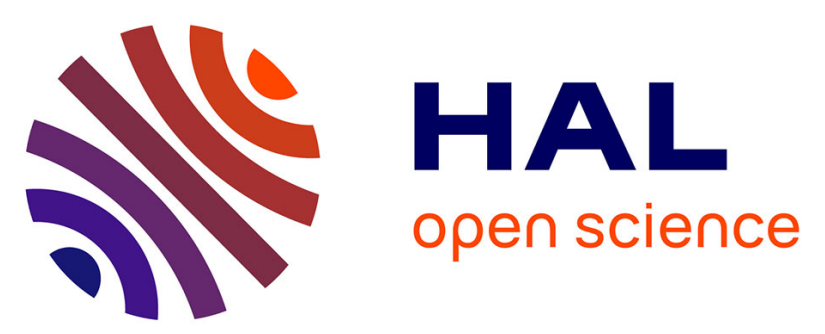

\title{
Efficient Stabilization of Advection Terms Involved in Separated Representations of Boltzmann and Fokker-Planck Equations
}

Francisco Chinesta, Emmanuelle Abisset-Chavanne, Amine Ammar, Elías Cueto

\section{To cite this version:}

Francisco Chinesta, Emmanuelle Abisset-Chavanne, Amine Ammar, Elías Cueto. Efficient Stabilization of Advection Terms Involved in Separated Representations of Boltzmann and FokkerPlanck Equations. Communications in Computational Physics, 2015, 17 (4), pp.975-1006. 10.4208/cicp.2014.m326 . hal-01206757

\section{HAL Id: hal-01206757 https://hal.science/hal-01206757}

Submitted on 29 Sep 2015

HAL is a multi-disciplinary open access archive for the deposit and dissemination of scientific research documents, whether they are published or not. The documents may come from teaching and research institutions in France or abroad, or from public or private research centers.
L'archive ouverte pluridisciplinaire HAL, est destinée au dépôt et à la diffusion de documents scientifiques de niveau recherche, publiés ou non, émanant des établissements d'enseignement et de recherche français ou étrangers, des laboratoires publics ou privés. 


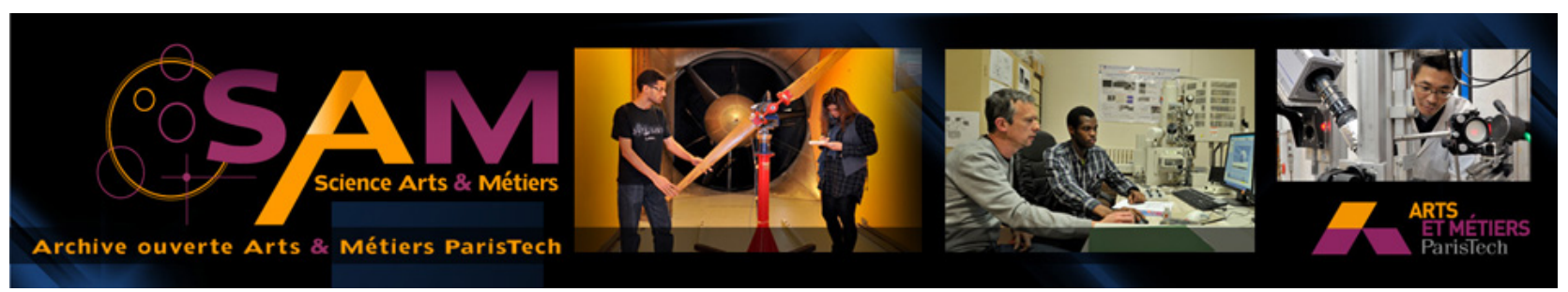

\section{Science Arts \& Métiers (SAM)}

is an open access repository that collects the work of Arts et Métiers ParisTech researchers and makes it freely available over the web where possible.

This is an author-deposited version published in: http://sam.ensam.eu

Handle ID: .http://hdl.handle.net/10985/10237

\section{To cite this version :}

Francisco CHINESTA, Emmanuelle ABISSET-CHAVANNE, Amine AMMAR, Elias CUETO Efficient Stabilization of Advection Terms Involved in Separated Representations of Boltzmann and Fokker-Planck Equations - Communications in Computational Physics - Vol. 17, nº 4, p.975$1006-2015$ 


\title{
Efficient Stabilization of Advection Terms Involved in Separated Representations of Boltzmann and Fokker-Planck Equations
}

\author{
F. Chinesta ${ }^{1, *}$, E. Abisset-Chavanne ${ }^{1}$, A. Ammar ${ }^{2}$ and E. Cueto ${ }^{3}$ \\ 1 GeM Institute, Ecole Centrale de Nantes, 1 rue de la Noe, BP 92101, F-44321 \\ Nantes cedex 3, France. \\ 2 Arts et Métiers ParisTech, 2 Boulevard du Ronceray, BP 93525, F-49035 Angers \\ cedex 01, France. \\ ${ }^{3}$ Aragon Institute of Engineering Research, Universidad de Zaragoza, Maria de \\ Luna, 7, E-50018 Zaragoza, Spain.
}

\begin{abstract}
The fine description of complex fluids can be carried out by describing the evolution of each individual constituent (e.g. each particle, each macromolecule, etc.). This procedure, despite its conceptual simplicity, involves many numerical issues, the most challenging one being that related to the computing time required to update the system configuration by describing all the interactions between the different individuals. Coarse grained approaches allow alleviating the just referred issue: the system is described by a distribution function providing the fraction of entities that at certain time and position have a particular conformation. Thus, mesoscale models involve many different coordinates, standard space and time, and different conformational coordinates whose number and nature depend on the particular system considered. Balance equation describing the evolution of such distribution function consists of an advection-diffusion partial differential equation defined in a high dimensional space. Standard mesh-based discretization techniques fail at solving high-dimensional models because of the curse of dimensionality. Recently the authors proposed an alternative route based on the use of separated representations. However, until now these approaches were unable to address the case of advection dominated models due to stabilization issues. In this paper this issue is revisited and efficient procedures for stabilizing the advection operators involved in the Boltzmann and Fokker-Planck equation within the PGD framework are proposed.
\end{abstract}

Key words: Boltzmann equation, Fokker-Planck equation, proper generalized decomposition, multidimensional models, convective stabilization. 


\section{Introduction}

The fine description of the behavior of a system composed by a series of microscopic entities, e.g. particles or molecules dispersed into a solvent, requires the consideration of all the entities as well as taking into account all existing interactions.

Despite the nowadays computational capabilities, the population of particles or macromolecules in a system of industrial interest is too large to be described in a discrete manner by considering all the involved individuals.

One possibility to reduce the size of the discrete models lies in considering only the particles of interest (that is, the suspended particles or the macromolecules), being the others (solvent particles) taken into account indirectly from their averaged effects on the particles of interest [26].

Thus for example, in the case of suspensions involving small rigid spherical particles, the motion equation of a particle whose position is described by $\mathbf{x}_{i}(t)$, is given by the Langevin's equation

$$
m \frac{d^{2} \mathbf{x}_{i}}{d t^{2}}=\xi\left(\frac{d \mathbf{x}_{i}}{d t}-\mathbf{V}\left(\mathbf{x}_{i}\right)\right)+\mathbf{F}_{i}^{\text {ext }}(t) ; \quad \forall i,
$$

where $m$ denotes the particle mass, $\mathbf{x}_{i}$ the position of particle $i, \xi$ the friction coefficient, $\mathbf{V}\left(\mathbf{x}_{i}\right)$ the fluid velocity at position $\mathbf{x}_{i}$ and $\mathbf{F}_{i}^{\text {ext }}(t)$ the other forces acting on particle $i$ (coming from an external potential or from solvent particles bombardment). We can notice that even if this model does not incorporate explicitly the solvent particles population, their effects are taken into account from the drift term $\xi\left(\frac{d \mathbf{x}_{i}}{d t}-\mathbf{V}\left(\mathbf{x}_{i}\right)\right)$ as well as by the impact forces of stochastic nature included into the term $\mathbf{F}_{i}^{\text {ext }}(t)$.

In the case of inertialess particles this description was traditionally substituted by continuous approaches involving the macroscopic field $C(\mathbf{x}, t)$ given the number of particles per unit of volume at position $\mathbf{x}$ and time $t$. The balance equation results in this case the classical advection-diffusion equation

$$
\frac{\partial C}{\partial t}+\mathbf{V} \cdot \nabla C=\nabla \cdot(\mathbf{D} \nabla C)+\mathcal{S}(\mathbf{x}, t)
$$

where the diffusion term involving the diffusivity tensor $\mathbf{D}$ is related to the stochastic interaction effects, $\mathbf{V}$ is the medium velocity and $\mathcal{S}(\mathbf{x}, t)$ an eventual source term. Now, standard discretization techniques can be applied for solving the resulting transient 3D advection-diffusion equation (e.g. finite differences or finite elements among many other possibilities). Since the use of discretization techniques applying on the continuous partial differential equation requires the use of a mesh of the domain in which the model is defined, and then the solution of a linear system (many in the case of transient nonlinear models) in order to calculate the solution at any node of the mesh or grid, as soon as the domain becomes too large, the solution of such linear systems can become a real bottleneck. To circumvent this issue an alternative is to come back to the stochastic discrete approach that proceeds by tracking a large enough population of particles, whose path- 
lines result from two contributions, a drift related to $\mathbf{V}(\mathbf{x}, t)$ and a random contribution that follows adequate statistics.

In the general case involving inertial particles the system can be described by considering the distribution function $f(\mathbf{x}, \mathbf{v}, t)$, that in this case represents the number of particles per unit volume that, at position $\mathbf{x}$ and time $t$, have a velocity given by $\mathbf{v}$. Note that $\mathbf{v}$ is a conformational coordinate because now the particles velocity does not coincide with the medium velocity $\mathbf{V}$ and at each position and time, particles have a velocity distribution that evolves in space and time.

Thus, the introduction of conformational coordinates in the system description involves the increase of the model dimensionality. In what follows we consider two kind of models, Boltzmann and Fokker-Planck models.

\subsection{The Vlasov-Poisson-Boltzmann equation}

We consider the dynamics of $P$ electrically charged particles of mass $m$. When $P$ becomes too large, direct molecular dynamics simulations result prohibitive from the computing time viewpoint. Thus, more than describing the system from the position and velocities of all the particles, one could introduce the function $f(t, \mathbf{x}, \mathbf{v})$ given the number of particles that at time $t$, are located within an elementary volume $d \mathbf{x}=(d x, d y, d z)$ placed at position $\mathbf{x}$ and having velocities within the volume defined by $d \mathbf{v}=(d u, d v, d w)$ around $\mathbf{v}$. Thus, the density balance writes

$$
\frac{\partial f}{\partial t}+\mathbf{v} \cdot \nabla_{x} f+\mathbf{a}(\mathbf{x}, t) \cdot \nabla_{v} f=\mathcal{S}(t, \mathbf{x}, \mathbf{v}),
$$

where $\nabla_{x}$ and $\nabla_{v}$ represent the gradient operator in the physical and velocity spaces respectively. It is here assumed that the acceleration $\mathbf{a}=\frac{d \mathbf{v}}{d t}$ does not depend on the velocity (by this reason it is not affected by the differential operator $\left.\nabla_{v}\right)$. The source term $\mathcal{S}(t, \mathbf{x}, \mathbf{v})$ represents the so-called collision term that can be defined from an appropriate physical modeling [53].

We do not need any physics to model the velocity field $\mathbf{v}$ because now it is a real coordinate, like the spatial ones. On the contrary, we need to define the acceleration field $\mathbf{a}(\mathbf{x}, t)$. For this purpose we consider Newton's law $\mathbf{a}=\frac{\mathbf{F}}{m}$, and compute the force acting on the particles by taking into account the nature of the system, that in the case here addressed consists of a population of charged particles interacting by means of Coulomb's potential. Thus the electrostatic potential $U(\mathbf{x}, t)$ depends on the particles spatial distribution that can be described from the marginal distribution $\bar{f}(\mathbf{x}, t)$

$$
\bar{f}(\mathbf{x}, t)=\int_{\mathbb{R}^{3}} f(\mathbf{x}, \mathbf{v}, t) d \mathbf{v} .
$$

Remark 1.1. 1. When particles are not charged the steady state solution of Eq. (1.3) leads to the Maxwell-Boltzmann distribution when the appropriate choice of the collision term describing the particles collisions is made. 
2. When the acceleration does not depend on the velocity (as was assumed in Eq. (1.3)) and the collision terms vanishes, an equivalence between the conservation equation and Liouville's theorem in the phase space can be established.

3. In general, modeling collision mechanisms is quite difficult. To circumvent this difficulty and assuming that the equilibrium distribution is known $f_{e q}(\mathbf{x}, \mathbf{v}, t)$, one could approximate the collision term by

$$
\mathcal{S}(t, \mathbf{x}, \mathbf{v})=\frac{f_{e q}(\mathbf{x}, \mathbf{v}, t)-f(\mathbf{x}, \mathbf{v}, t)}{\tau}
$$

where $\tau$ represents a relaxation time. This approximation leads to the so-called BGK models [10].

4. The kinetic theory formalism allows transforming a discrete model into its continuous counterpart. However, in general, continuous descriptions involve highly multidimensional spaces and their evolutions are governed by hyperbolic non-linear partial differential equations. To solve this kind of models, appropriate stabilized solvers, able to proceed in highly multidimensional spaces, are needed.

In the previous paragraphs we introduced some ideas related to kinetic theory models of systems composed of inertial particles. We are introducing in the next paragraphs a model involving interialess particles whose description requires the consideration of conformational coordinates [35]. A simple case consists of a population of rod-like intertialess particles, whose distribution is given by $\psi(\mathbf{x}, \mathbf{p}, t)$ where $\mathbf{p}$ defines rod orientations.

\subsection{The Fokker-Planck formalism}

Consider a dilute suspension of rod-like particles (short fibers, nanofibers, functionalized carbon nanotubes or even rod-like molecules). The configuration distribution function (also known as orientation distribution function) gives the probability of finding the particle oriented along a given direction. Obviously, this function depends on the physical coordinates (space and time) as well as on the configuration coordinates, that taking into account the rigid character of the particles, are defined on the surface of the unit sphere. Thus, we can write $\psi(\mathbf{x}, t, \mathbf{p})$, where $\mathbf{x}$ defines the position of the rod center of mass, $t$ the time and $\mathbf{p}$ the unit vector defining the rod orientation. The evolution of the distribution function is given by the Fokker-Planck equation

$$
\frac{d \psi}{d t}=-\nabla_{p}(\dot{\mathbf{p}} \psi)
$$

where $d / d t$ represents the material derivative. The orientation distribution function must verify the normality condition

$$
\int \psi(\mathbf{p}) d \mathbf{p}=1
$$


In the case of dilute suspensions the particles rotary velocity can be obtained from the Jeffery's equation that for rod-like particles reads

$$
\dot{\mathbf{p}}^{J}=\nabla \mathbf{v} \mathbf{p}-\left(\mathbf{p}^{T} \nabla \mathbf{v} \mathbf{p}\right) \mathbf{p},
$$

where $\nabla \mathbf{v}$ is the gradient of velocity tensor associated with the fluid flow undisturbed by the presence of the suspended particles. When particle concentration increases particles interactions occur modifying the Jeffery's velocity. In these circumstances a term is added to the Jeffery's rotary velocity that tends to randomize the orientation distribution

$$
\dot{\mathbf{p}}=\nabla \mathbf{v} \mathbf{p}-\left(\mathbf{p}^{T} \nabla \mathbf{v} \mathbf{p}\right) \mathbf{p}-D_{r} \frac{\nabla_{p} \psi}{\psi}=\dot{\mathbf{p}}^{J}-D_{r} \frac{\nabla_{p} \psi}{\psi}
$$

that, when introduced into the Fokker-Planck equation, leads to

$$
\frac{d \psi}{d t}=-\nabla_{p}\left(\dot{\mathbf{p}}^{J} \psi\right)+\nabla_{p} \cdot\left(D_{r} \nabla_{p} \psi\right)
$$

where $\dot{\mathbf{p}}^{J}$ denotes the Jeffery's rotary velocity expressed from Eq. (1.9) and $D_{r}$ is a rotary diffusion coefficient that some authors assume depending on the flow intensity.

In complex flows simulations the solution of the Fokker-Planck equations (1.6) or (1.10) involves some numerical difficulties related to: (i) its multidimensional character, i.e. $\psi(\mathbf{x}, t, \mathbf{p})$; (ii) the geometrical complexity of the physical domain $\Omega$; (iii) its purely advective character in the physical space; and (iv) the advection effects in the conformation space. These difficulties will be addressed in Section 1.4.

\subsection{On the numerical solution of kinetic theory models}

As argued above, the kinetic theory formalism allows transforming a discrete model into its continuous counterpart. However, in general, continuous descriptions involve highly multidimensional spaces and their evolutions are governed by hyperbolic non-linear partial differential equations. To solve this kind of models, appropriate stabilized solvers, able to proceed in highly multidimensional spaces, are needed.

\subsubsection{Boltzmann's model}

When addressing the Boltzmann's model previous issues where efficiently addressed by considering the Lattice-Boltzmann Method (LBM) [31,53]. The use of the LBM provides many of the advantages of molecular dynamics, including clear physical pictures, easy implementation, and fully parallel algorithms. The basic premise for using this simplified kinetic-type method for macroscopic fluid flows is that the macroscopic dynamics of a fluid is the result of the collective behavior of many microscopic particles in the system and that the macroscopic dynamics is not sensitive to the underlying details in microscopic physics. The LBM ancestor is the lattice gas automata (see for example $[25,40]$ ) which is constructed as a simplified, fictitious molecular dynamic in which space, time, 
and particle velocities are all discrete. It consists of a regular lattice with particles residing on the nodes. Starting from an initial state, the configuration of particles at each time step evolves in two sequential sub-steps: (a) streaming, in which each particle moves to the nearest node in the direction of its velocity, and $(b)$ collision, which occurs when particles arriving at a node interact and change their velocity directions according to specified rules. The lattice Boltzmann method considers a number of velocity directions depending on the considered cell and a velocity value associated to each one of these directions allowing to reach the neighbor location in the considered time step.

The interested reader can refer to $[29,30,37,47]$ for some theoretical aspects related to the method and some specificities related to the mesh. The LBM method has been successfully extended to problems involving non-regular grid with local mesh refinement [51] and to flows around moving objects [50]. Aspects related to fluid flows and to the solution of the Navier-Stokes equations can be found in [13,32]. Other complex fluids flows have been addressed by using the LBM, as for example viscous [56] and multiphase flow [33,44], flows in porous media [55], or those involving colloidal suspension [36]. Recently its use was extended to non Newtonian fluids flows [24]. Many other aspects such as the ones related to boundary conditions, complex geometry, turbulence, have been extensively addressed [14].

\subsubsection{Fokker-Planck model}

Fokker-Planck formalism is used to describe the evolution of the configuration distribution function, which represents the fraction of individuals having a particular configuration at a space point and time. The first works concerning its resolution include the Brownian dynamics approach used in the CONNFESSIT approach [48]. This approach was considered in [52] for treating MBS (multi bead spring) models. A similar technique was proposed in [54] in the context of MBS kinetic models, which introduces a change of variable and uses a Monte-Carlo technique for treating the diffusion term. In these techniques a high number of particles are introduced in the stochastic simulation to account for Brownian effects. A multi-scale approach using deterministic particles for treating the advection and a different set of particles to account for diffusion effects, which leads in fact to a multi-scale approach, was considered in [34]. The same idea was used in the case of short fibre suspensions flows in [15]. In that work, the discretisation of the advection dominated Fokker-Planck equation governing the fibre orientation was carried out using a particle technique, where the diffusion term was modelled from random walks. It was pointed out that the number of fibres required in this stochastic simulation to describe the fibre distribution increases significantly with the diffusion coefficient. Thus, it was argued that for practical applications the use of the particle method in the framework of a stochastic simulation is restricted to very slight diffusion effects. Another deterministic particle approach, very close to that proposed in [11], was analyzed in [3] using smooth particles, but it was noticed that the impact of smoothing on the solution can be significant. Moreover, the fact that the Fokker-Planck equation is defined in a multidimensional space induces the necessity of using an extremely large number of particles, 
with the associate unfavorable incidence on the methods efficiency. The combination of Brownian dynamics with a macroscopic treatment of motion equations is at the basis of the micro-macro approaches deeply reviewed in [35].

When diffusion effects vary in a large interval, continuous approximations using a fixed or moving mesh seem to be suitable. In this case accurate stabilizations are required for dealing with small diffusion effects. Some attempts at solving the FokkerPlanck equation using a fixed mesh discretisation exist $[12,39]$. The main difficulties in this approach are related to the multidimensional character of the problem. Thus, the linear systems obtained after usual implicit or semi-implicit space-time discretisations are extremely large for a practical inversion. On the other hand, explicit discretisations, which do not require matrix inversions, have the constraint of too small time steps.

The use of determinist mesh-based discretizations fails when addressing highly multidimensional Fokker-Planck equations and advanced techniques allowing circumventing the curse of dimensionality are needed.

\subsection{Circumventing curse of dimensionality by using separated representations}

As just illustrated, the description of complex systems within a mesoscopic framework requires in general the introduction of some (sometimes many) conformational coordinates, that implies the increase of the dimensionality of the associated balance equations. In these situations standard mesh-based discretization techniques fail because the curse of dimensionality. Consider a problem defined in a space of dimension $D$ for the unknown field $u\left(x_{1}, \cdots, x_{D}\right)$. Here, the coordinates $x_{i}$ denote any usual coordinate related to physical space, time, or conformation space, for example. In the simplest approach, we seek a solution for $\left(x_{1}, \cdots, x_{D}\right) \in \Omega_{1} \times \cdots \times \Omega_{D}$. A mesh based approach in which each coordinate involves $M$ nodes will imply the order of $M^{D}$ degrees of freedom. As soon as $D$ becomes moderately high the number of degrees of freedom is out of the nowadays computational capabilities.

Proper Generalized Decomposition - PGD - yields an approximate solution $u^{N}$ in the separated form

$$
u^{N}\left(x_{1}, \cdots, x_{D}\right)=\sum_{i=1}^{N} F_{i}^{1}\left(x_{1}\right) \cdot \ldots \cdot F_{i}^{D}\left(x_{D}\right)=\sum_{i=1}^{N} \prod_{j=1}^{D} F_{i}^{j}\left(x_{j}\right)
$$

where both the number of terms $N$ and the functions $F_{i}^{j}\left(x_{j}\right)$ are unknown a priori.

The representation (1.11) is similar to the classical separation of variables used for solving partial differential equations analytically (in the few instances when this is at all possible!). It is quite general in the sense that any polynomial can be expressed in that form. The main difference between (1.11) and a classical polynomial representation is that the functions $F_{i}^{j}\left(x_{j}\right)$, and not only their respective weights, are unknown a priori. 
The PGD approximation (1.11) is thus a sum of $N$ functional products involving each a number $D$ of functions $F_{i}^{j}\left(x_{j}\right)$ that are unknown a priori. It is constructed by successive enrichments, whereby each functional product is determined in sequence. At a particular enrichment step $n+1$, the functions $F_{i}^{j}\left(x_{j}\right)$ are known for $i \leq n$ from the previous steps, and one must compute the new product involving the $D$ unknown functions $F_{n+1}^{j}\left(x_{j}\right)$. This is achieved by invoking the weak form of the problem under consideration. The resulting discrete system is non-linear, which implies that iterations are needed at each enrichment step. A one-dimensional problem can thus be defined in $\Omega_{j}$ for each of the $D$ functions $F_{n+1}^{j}\left(x_{j}\right)$.

If $M$ nodes are used to discretize each coordinate space $\Omega_{j}$, the total number of PGD unknowns is $N \times M \times D$ instead of the $M^{D}$ degrees of freedom involved in standard meshbased discretizations. Moreover, all numerical experiments carried out to date with PGD show that the number of terms $N$ required to obtain an accurate solution is not a function of the problem dimension $D$, but it rather depends on the separable character of the exact solution. The PGD thus often avoids the exponential complexity with respect to the problem dimension.

Separated representations were successfully applied for solving the multidimensional Fokker-Planck equation describing complex fluids in the framework of kinetic theory. In [4], we addressed the solution of the linear Fokker-Planck equation describing multibead-spring molecular models of polymer solutions in steady-state homogeneous flows. The solution procedure was extended to non-linear kinetic theory descriptions of complex fluids in [45]. The transient case was addressed in [5]. A deeper analysis of nonlinear and transient models was considered in [7]. Extension of these developments to the simulation of complex flows was performed in $[2,46,49]$, thus opening very encouraging perspectives and claiming the necessity of defining efficient stabilization techniques. Finally, PGD was implemented in [16] to solve the stochastic equation within the framework of Brownian Configuration Fields. Models involving suspensions and colloidal systems were considered in $[1,22,27,28,41,43]$ and kinetic descriptions of microstructural mixing in $[17,42]$. The interested reader can consult [19] for an exhaustive overview of PGD applications in computational rheology and the more general reviews [18,20,21].

Multidimensional models encountered in the finer descriptions of matter (ranging from quantum chemistry to statistical mechanics) were revisited in [6]. The multidimensional chemical master equation was efficiently solved in [9]. Finally Langer's equation governing phase transitions was considered in [38].

\section{Stabilized discrete form of the Boltzmann's advection operator}

Separated representations involved in Proper Generalized Decomposition strategies allow circumventing the curse of dimensionality, but when they are applied for solving advection problems, the issue related to the advective stabilization must be carefully ad- 
dressed.

In what follows we consider the advection term in the Boltzmann equation (1.3) that leads to the simplified steady-state transport equation

$$
\mathbf{v} \cdot \nabla_{x} f=0
$$

that includes two major numerical difficulties: (i) it is multidimensional since $f=f(\mathbf{x}, \mathbf{v})$ and (ii) it is purely advective. Note that $\mathbf{v}$ in the present case is a coordinate of the model, a configurational one, because at each position $\mathbf{x}$ it is assumed the existence of many particles with different velocities $\mathbf{v}$.

In what follows and without loss of generality we are considering a one dimensional space that implies $f(x, v), x \in \Omega_{x} \subset \mathbb{R}$ and $v \in \Omega_{v} \subset \mathbb{R}$. Even if $\Omega_{v}$ could be the whole real axis, i.e. $\Omega_{v}=(-\infty, \infty)$ in gas kinetics, here we assume a large enough finite interval ensuring that the solution is fully contained inside. In the present case, because of the null collision term, this hypothesis fully applies as soon as the boundary condition involves velocities defined in a finite interval.

In the one-dimensional case Eq. (2.1) reads

$$
v \frac{\partial f}{\partial x}=0 \text { in } \Omega_{x} \times \Omega_{v}
$$

with $\Omega_{x}=(0, L)$ and $\Omega_{v}=[-U, U], U \neq \infty$.

The advective character of Eq. (2.2) requires enforcing boundary condition on $\Gamma_{l}$ and $\Gamma_{r}, \Gamma_{l}=\left(x=0, y \in \Omega_{v}^{+}\right)$and $\Gamma_{r}=\left(x=L, v \in \Omega_{v}^{-}\right)$, being $\Omega_{v}^{+}=[0, U]$ and $\Omega_{v}^{-}=[-U, 0]$.

\subsection{A "naive" PGD-based discretization}

The solution is searched by considering the simplest and more intuitive separated representation of $f(x, v)$

$$
f(x, v) \approx \sum_{i=1}^{i=N} X_{i}(x) V_{i}(v) .
$$

In order to build-up such a separated representation we proceed iteratively. If we assume that the $n-1$ terms of the finite sum (2.3) have been already calculated $(n \leq N)$, at iteration $n$ we should calculate $X_{n}(x)$ and $V_{n}(v)$. For that purpose we are considering the simplest strategy, the alternated directions fixed point algorithm widely considered in our former works, that proceeds by assuming $V_{n}$ known when calculating $X_{n}$ and then updating $V_{n}$ from the just calculated $X_{n}$. The process continues until reaching the convergence, that is, the fixed point of the nonlinear iteration. As soon as the new term of the finite sum (2.3), $X_{n}(x) \cdot V_{n}(v)$ has been obtained, the solution accuracy is checked by using an appropriate error estimator [8] and if the associated error is not small enough a new couple of functions $X_{n+1}(x)$ and $V_{n+1}(v)$ will be calculated. In what follows we are illustrating both steps, the one associated with the calculation of $X_{n}(x)$ and the one related to $V_{n}(v)$. 
1. By assuming $V_{n}(v)$ known (it is chosen randomly when starting the nonlinear iteration loop) the weak form related to Eq. (2.2) writes:

$$
\int_{\Omega_{x} \times \Omega_{v}} X^{*} V_{n} v \frac{d X_{n}}{d x} V_{n} d x d v=-\int_{\Omega_{x} \times \Omega_{v}} X^{*} V_{n} v \sum_{i=1}^{n-1}\left(\frac{d X_{i}}{d x} V_{i}\right) d x d v
$$

for all the test functions $X^{*}$ in an appropriate functional space and where the dependence of $X_{i}$ and $V_{i}$ on the coordinates $x$ and $v$ respectively was omitted for the sake of clarity.

Since all the functions involving the velocity coordinate $v$ are known, by integrating in $\Omega_{v}$, Eq. (2.4) reduces to:

$$
\int_{\Omega_{x}} X^{*} \alpha \frac{d X_{n}}{d x} d x=-\int_{\Omega_{x}} X^{*} \sum_{i=1}^{n-1}\left(\beta_{i} \frac{d X_{i}}{d x}\right) d x
$$

where coefficients $\alpha$ and $\beta_{i}$ are given by:

$$
\alpha=\int_{\Omega_{v}} V_{n}^{2} v d v
$$

and

$$
\beta_{i}=\int_{\Omega_{v}} V_{n} V_{i} v d v
$$

respectively.

The right hand member involves a function of $x$

$$
g(x)=\sum_{i=1}^{n-1} \beta_{i} \frac{d X_{i}}{d x}
$$

involving in turn functions that were calculated previously.

The strong form related to Eq. (2.5) reads

$$
\alpha \frac{d X_{n}}{d x}=-g(x)
$$

an equation that could be integrated from the boundary conditions by using an appropriate upwinding depending on the sign of $\alpha$. We will come back to this issue later.

2. When considering the just computed $X_{n}(x)$ the weak form related to Eq. (2.2) writes:

$$
\int_{\Omega_{x} \times \Omega_{v}} X_{n} V^{*} v \frac{d X_{n}}{d x} V_{n} d x d v=-\int_{\Omega_{x} \times \Omega_{v}} X_{n} V^{*} v \sum_{i=1}^{n-1}\left(\frac{d X_{i}}{d x} V_{i}\right) d x d v \text {. }
$$


Since all functions involving the space coordinate $x$ are known, by integrating in $\Omega_{x}$, Eq. (2.10) reduces to:

$$
\int_{\Omega_{v}} V^{*} \gamma v V_{n} d v=-\int_{\Omega_{v}} V^{*} \sum_{i=1}^{n-1}\left(\xi_{i} v V_{i}\right) d v,
$$

where coefficients $\gamma$ and $\delta_{i}$ are given by:

$$
\gamma=\int_{\Omega_{x}} X_{n} \frac{d X_{n}}{d x} d x
$$

and

$$
\xi_{i}=\int_{\Omega_{x}} X_{n} \frac{d X_{i}}{d x} d x
$$

respectively.

The right hand member involves a function of $v$

$$
h(v)=\sum_{i=1}^{n-1} \xi_{i} v V_{i}
$$

involving functions that were calculated previously.

The strong form related to Eq. (2.11) reads

$$
v V_{n}=-h(v)
$$

equation that is purely algebraic.

The just described numerical scheme fails to construct the separated representation. We are analyzing the possible reasons of this lack of convergence. First of all we should discuss the way of prescribing boundary conditions. We assume that on $\Gamma_{l}$ the solution is $f_{l}(v)$ and that on $\Gamma_{r}$ the solution is given by $f_{r}(v)$.

When considering the separated representation constructor just described, the usual way to enforce Dirichlet boundary conditions consists of choosing some couples of separated functions regular enough and that verifies the boundary conditions, and add those terms to the first terms of the separated representation of $f(x, y)$.

The simplest choice in the case here addressed consists of considering functions $X_{1}(x)$, $V_{1}(v), X_{2}(x)$ and $V_{2}(v)$ such that $X_{1} \cdot V_{1}+X_{2} \cdot V_{2}$ ensures the verification of boundary conditions on both boundaries $\Gamma_{l}$ and $\Gamma_{r}$. We consider:

$$
\left\{\begin{array}{l}
X_{1}(x)=1-\frac{x}{L^{\prime}}, \\
V_{1}(v)= \begin{cases}f_{l}(v), & v \in \Omega_{v}^{+}, \\
0, & v \in \Omega_{v}^{-},\end{cases}
\end{array}\right.
$$


that allows enforcing the boundary condition on $\Gamma_{l}$ and

$$
\left\{\begin{array}{l}
X_{2}(x)=\frac{x}{L}, \\
V_{2}(v)= \begin{cases}0, & v \in \Omega_{v}^{+}, \\
f_{r}(v), & v \in \Omega_{v}^{-},\end{cases}
\end{array}\right.
$$

that prescribes the boundary condition on $\Gamma_{r}$.

Thus, since the first two terms of the finite sum ensures satisfying the Dirichlet boundary condition, all the other terms $n>2$ must vanish on the Dirichlet boundaries.

Physically, advection applies in the $x$-direction in $\Omega_{v}^{+}$and in the opposite direction in $\Omega_{v}^{-}$, however the separated representation constructor only consider an "averaged" one when integrating Eq. (2.9):

$$
\begin{cases}X_{n}(x=0)=0, & \alpha \geq 0, \\ X_{n}(x=L)=0, & \alpha \leq 0 .\end{cases}
$$

Enforcing zero is only well defined in $\Omega_{x} \times \Omega_{v}^{+}$when $\alpha \geq 0$ and in $\Omega_{x} \times \Omega_{v}^{-}$when $\alpha \leq 0$. However the separated representation constructor cannot differentiate these cases because there is no information on the velocity when solving the problem involving the space coordinate (2.9).

\subsection{Enhanced PGD-based discretization}

The discussion that we just addressed should be very explicit for people using LatticeBoltzmann (LB) strategies. It is well known that LB strategies consist of two steps, the so-called streaming that applies convection and the so-called collision that include all the other mechanisms. In the streaming step, the nodal field is transported to the neighboring nodes, and it differentiates each direction from its opposite in order to perform an exact integration along the characteristics (exact because the nodal spacing and the velocities are chosen in order to jump from each node to the neighboring nodes simultaneously at each time step). In our case the situation is a bit more complex because we are considering not only some directions and a unique velocity value associated to each one of these directions, but any direction (in 2D or 3D) and all the velocities in the interval $\Omega_{v}$. However, inspired by the Lattice-Boltzmann procedures, we consider the enhanced conformational space $\left(x, s, v^{+}\right) \in\left(\Omega_{x} \times \Omega_{s} \times \Omega_{v}^{+}\right)$, where $s$ represents the velocity sign, $v^{+}=|v|$ and $\Omega_{s}=\{-1,1\}$. The associated separated representation writes:

$$
f\left(x, s, v^{+}\right) \approx \sum_{i=1}^{i=N} X_{i}(x, s) V_{i}\left(v^{+}\right),
$$

where again inspired from LB techniques the space and the sign are maintained coupled. In what follows we are analyzing how this alternative representation modifies the 
problem in space. By assuming $V_{n}\left(v^{+}\right)$known (it is chosen randomly when starting the nonlinear iteration loop) the weak form related to Eq. (2.2) writes:

$$
\begin{aligned}
& \int_{\Omega_{x} \times \Omega_{s} \times \Omega_{v}^{+}} X^{*} V_{n} s v^{+} \frac{d X_{n}}{d x} V_{n} d x d s d v^{+} \\
= & -\int_{\Omega_{x} \times \Omega_{s} \times \Omega_{v}^{+}} X^{*} V_{n} s v^{+} \sum_{i=1}^{n-1}\left(\frac{d X_{i}}{d x} V_{i}\right) d x d s d v^{+} .
\end{aligned}
$$

Remark 2.1. In this expression the integral involving the sign coordinate $s$ is not meaningful because this coordinate is discrete. In this case the integral on the parametric space $s$ must be read as a sum for the two possible values $s= \pm 1$.

Now as all the functions involving the velocity coordinate $v$ are known, by integrating in $\Omega_{v}$, Eq. (2.4) reduces to:

$$
\int_{\Omega_{x} \times \Omega_{s}} X^{*} \alpha s \frac{d X_{n}}{d x} d x d s=-\int_{\Omega_{x} \times \Omega_{s}} X^{*} s \sum_{i=1}^{n-1}\left(\beta_{i} \frac{d X_{i}}{d x}\right) d x d s,
$$

where coefficients $\alpha$ and $\beta_{i}$ are now given by

$$
\alpha=\int_{\Omega_{v}^{+}} V_{n}^{2} v^{+} d v^{+}
$$

and

$$
\beta_{i}=\int_{\Omega_{v}^{+}} V_{n} V_{i} v^{+} d v^{+}
$$

respectively. The associated strong form reads:

$$
\alpha s \frac{d X_{n}}{d x}=-\operatorname{sg}(x)
$$

that results in two independent equations, one for $s=1$ and the other for $s=-1$

$$
\left\{\begin{array}{cl}
\alpha \frac{d X_{n}}{d x}=-g(x), & X_{n}(x=0, s=1)=0 \\
-\alpha \frac{d X_{n}}{d x}=g(x), & X_{n}(x=L, s=-1)=0
\end{array}\right.
$$

that despite the fact of being formally identical both are integrated by using opposite upwinding and then each one considers the appropriate boundary conditions.

The calculation of $V_{n}\left(v^{+}\right)$does not implies any particularity. It is performed as described in Section 2.1. 


\section{Stabilized Fokker-Planck advection operators}

In this section we are focussing on the discrete stabilized form of the advection operator appearing in the Fokker-Planck equation that is more standard than the one involved in the Boltzmann equation in the sense that the advection field is now a given known field instead of a conformation coordinate.

For the sake of simplicity we consider the steady state 2D Fokker-Planck equation related to a dilute enough suspension of rod-like particles whose orientation distribution can be fully described by using the space $\mathbf{x}$ and orientation $\theta$ coordinates. Thus $\psi(\mathbf{x}, \theta)$ express the fraction of rods that at position $\mathbf{x}$ have an orientation $\theta$. The associated balance equation in absence of diffusion mechanics (the suspension is assumed dilute enough to neglect rods interactions) reads:

$$
\mathbf{v}(\mathbf{x}) \cdot \nabla \psi+\frac{\partial}{\partial \theta}(\dot{\theta} \psi)=0
$$

with $\mathbf{x} \in \Omega_{x}, \theta=\Omega_{\theta}=[0,2 \pi)$ and $\mathbf{v}(\mathbf{x})$ the velocity field that depends on the space coordinate $\mathbf{x}$.

It is important to note that the fact of neglecting diffusion mechanisms represents the worst scenario from a numerical point of view because when diffusion effects are large enough the integration of the Fokker-Planck equation does not require stabilization.

In the previous equation (3.1) the rotary velocity $\dot{\theta}$ comes from the Jeffery's equation particularized to $2 \mathrm{D}$ which implies $\mathbf{p}^{T}=(\cos \theta, \sin \theta)$. As Jeffery's equation (1.9) implies a dependence of the rotary velocity on the local gradient of velocity and the last one depends on the space coordinate $\mathbf{x}$, we can finally write $\dot{\theta}=\dot{\theta}(\mathbf{x}, \theta)$. Thus depending on the point $\mathbf{x}$ and the orientation $\theta$ the resulting rotary velocity can be positive $\dot{\theta} \geq 0$ or negative $\dot{\theta} \leq 0$, and this fact, far to be irrelevant or transparent from a computational point of view, determines the stabilization schema to be considered for integrating the term involving the angular derivative of $(\dot{\theta} \psi)$.

Eq. (3.1) involves two major numerical difficulties, the first one related to its highdimensionality (the distribution $\psi$ and the rotary velocity $\dot{\theta}$ depend on both the space and angular coordinates $\mathbf{x}$ and $\theta$ respectively). The second, and probably the most important, lies in the fact that the sign of $\dot{\theta}$ also depends on both coordinates ( $\mathbf{x}$ and $\theta$ ) determining the advective stabilization to be considered.

The first issue, the one related to the dimensionality is not a major issue when we proceed within a separated representation framework as it is the case when using the proper generalized decomposition. Within this framework we can assume the standard separated representation of the distribution function $\psi(\mathbf{x}, \theta)$

$$
\psi(\mathbf{x}, \theta) \approx \sum_{i=1}^{N} X_{i}(\mathbf{x}) \cdot \Theta_{i}(\theta)
$$

involving $N$ terms, each one consisting of a function of the space coordinate $\mathbf{x}$ and other depending the angular coordinate $\theta, X_{i}$ and $\Theta_{i}$ respectively. All these functions are a 
priori unknown and they will be calculated by invoking the PGD constructor detailed later.

Concerning the separated representation of $\dot{\theta}(\mathbf{x}, \theta)$, being a fully known field, it can be performed by applying a singular value decomposition (SVD) or in the higher multidimensional cases, its multidimensional counterpart, the so-called high-order singular value decomposition (HOSVD). Thus, it results

$$
\dot{\theta}(\mathbf{x}, \theta) \approx \sum_{i=1}^{N_{\theta}} F_{i}(\mathbf{x}) G_{i}(\theta) .
$$

The second issue, the one related to the stabilized discrete form of the advection operator applying in the angular coordinate, is more delicate. As pointed out above the rotary velocity $\dot{\theta}$ depends on $\mathbf{x}$ and then one could expect that depending on the point $\mathbf{x}$ the advection term

$$
\mathcal{A}=\frac{\partial}{\partial \theta}(\dot{\theta} \psi)
$$

should be integrated by using an upwinding stabilization depending on the sign of $\dot{\theta}$ at that position. However, within the "naive" implementation of the PGD constructor when solving the angular problem all the space information appears in a global form (functions depending on the $\mathbf{x}$ coordinate were integrated in $\Omega_{x}$ ) and then the same stabilization is used everywhere. This issue is in fact very similar to the one just discussed in the previous section when we analyzed the "naive" implementation of the PGD for discretizing the Boltzmann advection operator.

\subsection{Enhanced PGD-based discretization}

As we just mentioned the rotary velocity $\dot{\theta}(\mathbf{x}, \theta)$ is fully defined in $\Omega_{x} \times \Omega_{\theta}$ and its sign will determine the upwind stabilization of its discrete counterpart. For this purpose we consider the decomposition implying the positive and negative parts of $\dot{\theta}$ according to:

$$
\left\{\begin{array}{l}
\dot{\theta}^{+}(\mathbf{x}, \theta)=\max \{\dot{\theta}(\mathbf{x}, \theta), 0\}, \\
\dot{\theta}^{-}(\mathbf{x}, \theta)=\min \{\dot{\theta}(\mathbf{x}, \theta), 0\},
\end{array}\right.
$$

and the associated separated representations

$$
\dot{\theta}^{+}(\mathbf{x}, \theta) \approx \sum_{i=1}^{N_{\theta}^{+}} F_{i}^{+}(\mathbf{x}) G_{i}^{+}(\theta),
$$

and

$$
\dot{\theta}^{-}(\mathbf{x}, \theta) \approx \sum_{i=1}^{N_{\theta}^{-}} F_{i}^{-}(\mathbf{x}) G_{i}^{-}(\theta)
$$

respectively. 
Eq. (3.1) is now rewritten by considering the decomposition $\dot{\theta}=\dot{\theta}^{+}+\dot{\theta}^{-}$:

$$
\mathbf{v}(\mathbf{x}) \cdot \nabla \psi+\frac{\partial}{\partial \theta}\left(\dot{\theta}^{+} \psi\right)+\frac{\partial}{\partial \theta}\left(\dot{\theta}^{-} \psi\right)=0
$$

By introducing the separated representations (3.6) and (3.7) into the Fokker-Planck equation (3.8) and using appropriate upwinding stabilizations of both advective terms involving the angular coordinate, according to the rotary velocity sign, stable solutions can be calculated within the PGD framework.

We are illustrating one enrichment step of the algorithm. For the sake of notational simplicity, and of course, without loss of generality, we assume that both $N_{\theta}^{+}$and $N_{\theta}^{-}$ consist of a unique term, that is:

$$
\dot{\theta}^{+}(\mathbf{x}, \theta) \approx F^{+}(\mathbf{x}) G^{+}(\theta)
$$

and

$$
\dot{\theta}^{-}(\mathbf{x}, \theta) \approx F^{-}(\mathbf{x}) G^{-}(\theta)
$$

implying

$$
\mathbf{v}(\mathbf{x}) \cdot \nabla \psi+\frac{\partial}{\partial \theta}\left(F^{+} G^{+} \psi\right)+\frac{\partial}{\partial \theta}\left(F^{-} G^{-} \psi\right)=0 .
$$

The Fokker-Planck weak form reads:

$$
\int_{\Omega_{x} \times \Omega_{\theta}} \psi^{*}\left(\mathbf{v}(\mathbf{x}) \cdot \nabla \psi+\frac{\partial}{\partial \theta}\left(F^{+} G^{+} \psi\right)+\frac{\partial}{\partial \theta}\left(F^{-} G^{-} \psi\right)\right) d \mathbf{x} d \theta=0 .
$$

At the enrichment step $n(n<N)$, the distribution function $\psi^{n-1}$ consists of

$$
\psi^{n-1}(\mathbf{x}, \theta)=\sum_{i=1}^{n-1} X_{i}(\mathbf{x}) \Theta_{i}(\theta),
$$

where functions $X_{i}$ and $\Theta_{i}$ were calculated in the previous enrichment steps. Now, we would like to compute $\psi^{n}$ according to

$$
\psi^{n}(\mathbf{x}, \theta)=\sum_{i=1}^{n-1} X_{i}(\mathbf{x}) \Theta_{i}(\theta)+X_{n}(\mathbf{x}) \Theta_{n}(\theta)=\psi^{n-1}+X_{n}(\mathbf{x}) \Theta_{n}(\theta),
$$

that implies the test function choice $\psi^{*}$

$$
\psi^{*}=X^{*} \Theta_{n}+X_{n} \Theta^{*} .
$$

Introducing the trial and test functions (3.14) and (3.15) into the problem weak form (3.12) we obtain a nonlinear problem involving the unknown functions $X_{n}$ and $\Theta_{n}$. The simplest linearization strategy consists of a fixed point alternated directions scheme that proceeds by calculating $X_{n}$ assuming $\Theta_{n}$ known, and then updating $\Theta_{n}$ from the just calculated $X_{n}$. The process continues until reaching convergence, that is, the fixed point. In what follows we explicit both steps. 
1. When assuming $\Theta_{n}$ known, $\Theta^{*}$ vanishes and the weak form involving the unknown function $X_{n}$ writes:

$$
\begin{aligned}
& \int_{\Omega_{x} \times \Omega_{\theta}} X^{*} \Theta_{n}\left(\mathbf{v}(\mathbf{x}) \cdot \nabla\left(X_{n} \Theta_{n}\right)+\frac{\partial}{\partial \theta}\left(F^{+} G^{+} X_{n} \Theta_{n}\right)+\frac{\partial}{\partial \theta}\left(F^{-} G^{-} X_{n} \Theta_{n}\right)\right) d \mathbf{x} d \theta \\
= & -\int_{\Omega_{x} \times \Omega_{\theta}} X^{*} \Theta_{n} \mathbf{v}(\mathbf{x}) \cdot \nabla\left(\sum_{i=1}^{n-1} X_{i} \Theta_{i}\right) d \mathbf{x} d \theta \\
& -\int_{\Omega_{x} \times \Omega_{\theta}} X^{*} \Theta_{n}\left(\frac{\partial}{\partial \theta}\left(F^{+} G^{+}\left(\sum_{i=1}^{n-1} X_{i} \Theta_{i}\right)\right)+\frac{\partial}{\partial \theta}\left(F^{-} G^{-}\left(\sum_{i=1}^{n-1} X_{i} \Theta_{i}\right)\right)\right) d \mathbf{x} d \theta .
\end{aligned}
$$

Being all the functions depending in the angular coordinate $\theta$ known, Eq. (3.16) can be integrated in $\Omega_{\theta}$ leading to:

$$
\int_{\Omega_{x}} X^{*}\left(\alpha \mathbf{v}(\mathbf{x}) \cdot \nabla X_{n}+\beta F^{+} X_{n}+\gamma F^{-} X_{n}\right) d \mathbf{x}=\int_{\Omega_{x}} X^{*} f(\mathbf{x}) d \mathbf{x},
$$

where

$$
\begin{aligned}
& \alpha=\int_{\Omega_{\theta}} \Theta_{n}^{2} d \theta, \\
& \beta=\int_{\Omega_{\theta}} \Theta_{n} \frac{d}{d \theta}\left(G^{+} \Theta_{n}\right) d \theta, \\
& \gamma=\int_{\Omega_{\theta}} \Theta_{n} \frac{d}{d \theta}\left(G^{-} \Theta_{n}\right) d \theta,
\end{aligned}
$$

and $f(\mathbf{x})$ comes from the integration in $\Omega_{\theta}$ of the right hand member of Eq. (3.16) that only involves known functions.

The strong form related to Eq. (3.17) reads

$$
\alpha \mathbf{v}(\mathbf{x}) \cdot \nabla X_{n}+\beta F^{+} X_{n}+\gamma F^{-} X_{n}=f(\mathbf{x}) .
$$

Appropriate stabilized discretization (upwinding) techniques can be applied to both the weak (3.17) and the strong (3.21) forms.

2. When assuming $X_{n}$ known, $X^{*}$ vanishes and the weak form involving the unknown function $\Theta_{n}$ writes:

$$
\begin{aligned}
& \int_{\Omega_{x} \times \Omega_{\theta}} X_{n} \Theta^{*}\left(\mathbf{v}(\mathbf{x}) \cdot \nabla\left(X_{n} \Theta_{n}\right)+\frac{\partial}{\partial \theta}\left(F^{+} G^{+} X_{n} \Theta_{n}\right)+\frac{\partial}{\partial \theta}\left(F^{-} G^{-} X_{n} \Theta_{n}\right)\right) d \mathbf{x} d \theta \\
= & -\int_{\Omega_{x} \times \Omega_{\theta}} X_{n} \Theta^{*} \mathbf{v}(\mathbf{x}) \cdot \nabla\left(\sum_{i=1}^{n-1} X_{i} \Theta_{i}\right) d \mathbf{x} d \theta \\
& -\int_{\Omega_{x} \times \Omega_{\theta}} X_{n} \Theta^{*}\left(\frac{\partial}{\partial \theta}\left(F^{+} G^{+}\left(\sum_{i=1}^{n-1} X_{i} \Theta_{i}\right)\right)+\frac{\partial}{\partial \theta}\left(F^{-} G^{-}\left(\sum_{i=1}^{n-1} X_{i} \Theta_{i}\right)\right)\right) d \mathbf{x} d \theta .
\end{aligned}
$$


Being all the functions depending in the space coordinate $\mathbf{x}$ known, Eq. (3.22) can be integrated in $\Omega_{x}$ leading to:

$$
\int_{\Omega_{\theta}} \Theta^{*}\left(\chi \Theta_{n}+\xi \frac{d}{d \theta}\left(G^{+} \Theta_{n}\right)+\mu \frac{d}{d \theta}\left(G^{-} \Theta_{n}\right)\right) d \theta=\int_{\Omega_{\theta}} \Theta^{*} g(\theta) d \theta,
$$

where

$$
\begin{aligned}
& \chi=\int_{\Omega_{x}} X_{n} \mathbf{v}(\mathbf{x}) \cdot \nabla X_{n} d \mathbf{x}, \\
& \xi=\int_{\Omega_{x}} X_{n}^{2} F^{+} d \mathbf{x}, \\
& \mu=\int_{\Omega_{x}} X_{n}^{2} F^{-} d \mathbf{x},
\end{aligned}
$$

and $g(\theta)$ comes from the integration in $\Omega_{x}$ of the right hand member of Eq. (3.22) that only involves known functions.

The strong form related to Eq. (3.23) reads:

$$
\chi \Theta_{n}+\xi \frac{d}{d \theta}\left(G^{+} \Theta_{n}\right)+\mu \frac{d}{d \theta}\left(G^{-} \Theta_{n}\right)=g(\theta) .
$$

Appropriate stabilized (upwinding) discretization techniques can be applied to both the weak (3.23) and the strong (3.27) forms.

\section{Numerical results}

\subsection{D steady-state collision-free Boltzmann equation}

First we consider the one-dimensional steady state Boltzmann equation

$$
v \frac{\partial f}{\partial x}=0 \quad \text { in } \Omega_{x} \times \Omega_{v}
$$

with $\Omega_{x}=(0, L)$ and $\Omega_{v}=[-U, U], L=4$ and $U=4$.

Dirichlet boundary conditions are enforced on $\Gamma_{l}$ and $\Gamma_{r}\left(\Gamma_{l}=\left(x=0, y \in \Omega_{v}^{+}\right)\right.$and $\Gamma_{r}=\left(x=L, v \in \Omega_{v}^{-}\right), \Omega_{v}^{+}=[0, U]$ and $\left.\Omega_{v}^{-}=[-U, 0]\right)$

$$
\left\{\begin{array}{l}
f\left(x=0, v \in \Omega_{v}^{+}\right)=f_{l}(v), \\
f\left(x=L, v \in \Omega_{v}^{-}\right)=f_{r}(v) .
\end{array}\right.
$$

Fig. 1 depicts function $f_{l}(v)$, a sort of Gaussian with compact support in $\Omega_{v}^{+}$. Boundary condition on $\Gamma_{r}$ was considered to be the symmetric of $f_{l}(v)$ with respect to the axis $v=0$, that is $f_{r}(-|v|)=f_{l}(v)$. 


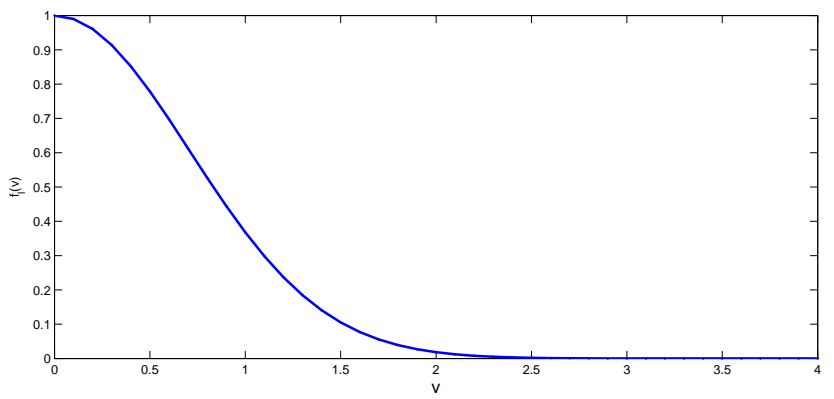

Figure 1: Boundary condition enforced on $\Gamma_{l}$.

In this case since the Boltzmann equation reduces to a pure advection, the exact solution of the problem $f^{e x}(x, v)$ reads

$$
f^{e x}(x, v)=\left\{\begin{array}{lll}
f_{l}(v), & x \in \Omega_{x}, & v \in \Omega_{v}^{+}, \\
f_{r}(v), & x \in \Omega_{x}, & v \in \Omega_{v}^{-} .
\end{array}\right.
$$

The solution is sought in the separated form

$$
f\left(x, s, v^{+}\right) \approx \sum_{i=1}^{i=N} X_{i}(x, s) V_{i}\left(v^{+}\right),
$$

and because the form of the exact solution (4.3), the optimal decomposition (separated representation) consists of a single term, that is $N^{o p}=1$.

As previously discussed, when considering the separated representation constructor, the usual way to enforce Dirichlet boundary conditions consists of choosing some couples of separated functions regular enough in order to verify boundary conditions, and add those terms to the first terms of the separated representation of $f(x, s)$. This choice is in principle arbitrary. Obviously if we consider

$$
\left\{\begin{array}{l}
X_{1}(x, s)=1 \\
V_{1}\left(v^{+}\right)=f_{l}(v),
\end{array}\right.
$$

the solution $f^{1}(x, v)=X_{1}(x, s) V_{1}\left(v^{+}\right)=f^{e x}(x, v)$ and then the enrichment process stops. The separated representation consists of a single term and it is then optimal.

To avoid such a super convergence we consider an initial guess that verifies the boundary condition without verifying the problem solution everywhere. To this purpose we consider the first term given by:

$$
\left\{\begin{array}{l}
X_{1}(x, s)= \begin{cases}1, & x=0, s=1, \\
1, & x=L, s=-1, \\
0, & \text { elsewhere }\end{cases} \\
V_{1}\left(v^{+}\right)=f_{l}(v) .
\end{array}\right.
$$




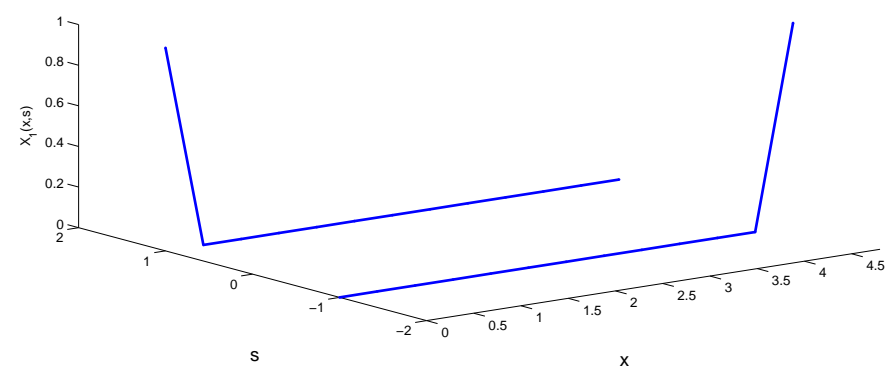

Figure 2: First space term of the separated representation $X_{1}(x, s)$. It allows enforcing the Dirichlet boundary condition but it does not represent the exact solution everywhere.

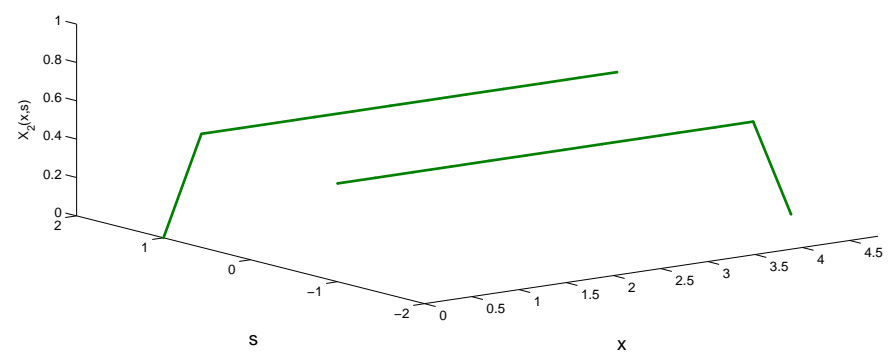

Figure 3: Space function $X_{2}(x, s)$ related to the second mode of the decomposition.

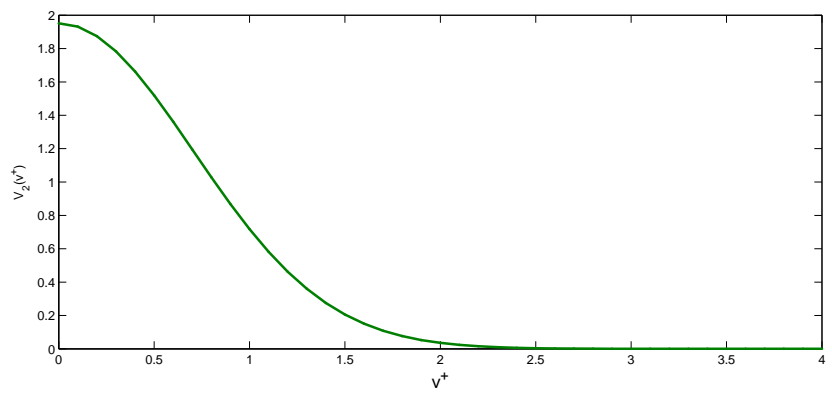

Figure 4: Velocity function $V_{2}\left(v^{+}\right)$related to the second mode of the decomposition.

The initial guess is depicted in Fig. 2. Because it only verifies the boundary conditions but not the solution in $\Omega_{x} \times \Omega_{v}$ one expects that the algorithm generates other terms (only one to ensure the most optimality).

Thus, from $f^{1}\left(x, s, v^{+}\right)=X_{1}(x, s) V_{1}\left(v^{+}\right)$the separated representation constructor calculate the second mode $X_{2}(x, s) V_{2}\left(v^{+}\right)$leading to the enriched solution $f^{2}\left(x, s, v^{+}\right)=$ $f^{1}\left(x, s, v^{+}\right)+X_{2}(x, s) V_{2}\left(v^{+}\right)$. Figs. 3 and 4 depict both functions $X_{2}(x, s)$ and $V_{2}\left(v^{+}\right)$respectively. As expected $f^{2}\left(x, s, v^{+}\right)$agrees in minute the exact solution and then the enrichment procedure stops and $f^{2}\left(x, s, v^{+}\right)$constitutes the searched solution. 


\subsection{D transient collision-free Boltzmann equation}

We consider now the transient solution of the collision-free Boltzmann equation

$$
\frac{\partial f}{\partial t}+v \frac{\partial f}{\partial x}=0
$$

defined in $\Omega_{x} \times \Omega_{v}, \Omega_{x}=(0, L)$ and $\Omega_{v}=[-U, U], U=1$ and $L=4$. The main difference is that now we must consider also the time coordinate defined in the time interval $\Omega_{t}=$ $(0,25]$.

Two separated representations where analyzed, the one considering

$$
f\left(x, t, s, v^{+}\right) \approx \sum_{i=1}^{i=N} X_{i}(x, s) T_{i}(t) V_{i}\left(v^{+}\right)
$$

and the one involving space-time functions

$$
f\left(x, t, s, v^{+}\right) \approx \sum_{i=1}^{i=\tilde{N}} X_{i}(x, t, s) V_{i}\left(v^{+}\right) .
$$

In the last case space-time functions $X_{i}(x, t, s)$ were calculated by using two different integrations. The first considering again a space-time separated representation, that is

$$
X_{i}(x, t, s) \approx \sum_{i=1}^{i=\hat{N}} R_{i}(x, s) S_{i}(t),
$$

leading to a sort of imbricated separated representation. The second possibility was the incremental integration of functions $X_{i}(x, t, s)$ by using an adequate solver for transient advection equations (e.g. streamline upwind scheme).

No significant differences were noticed when applying any of the just considered strategies. In what follows the scheme (4.8) is considered. First we consider the separated representation (4.8) and consider the following initial and boundary conditions:

$$
\left\{\begin{array}{l}
f\left(x \in \Omega_{x}, t=0, v \in \Omega_{v}\right)=0 \\
f\left(x=0, t, v \in \Omega_{v}^{+}\right)=f_{l}(v) \\
v f\left(x=L, t, v \in \Omega_{v}^{-}\right)=f_{r}(v)
\end{array}\right.
$$

where $f_{l}(v)$ is depicted in Fig. 5 (being $f_{r}(v)$ its symmetric image with respect to the axis $v=0$ ).

Figs. 6, 7 and 8 depict the seven most important function $X_{i}(x, s), T_{i}(t)$ and $V_{i}\left(v^{+}\right), i=$ $1, \cdots, 7$, respectively (without considering the functions used for enforcing the boundary conditions), and Fig. 9 different snapshots of the solution $f(x, t, v)$ at different times. The computation of this rich solution (transient multidimensional) is obtained in few seconds thanks to the separated representation features. 


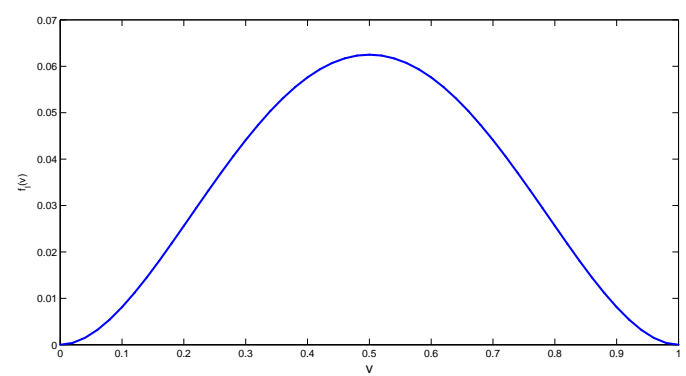

Figure 5: Dirichlet boundary condition $f_{l}(v)$ considered in the transient Boltzmann problem.

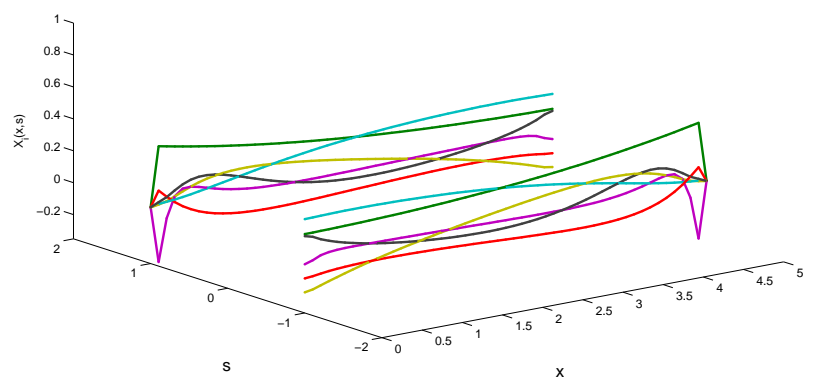

Figure 6: Seven most significant functions $X_{i}(x, s)$ involved in the Boltzmann solution separated representation.

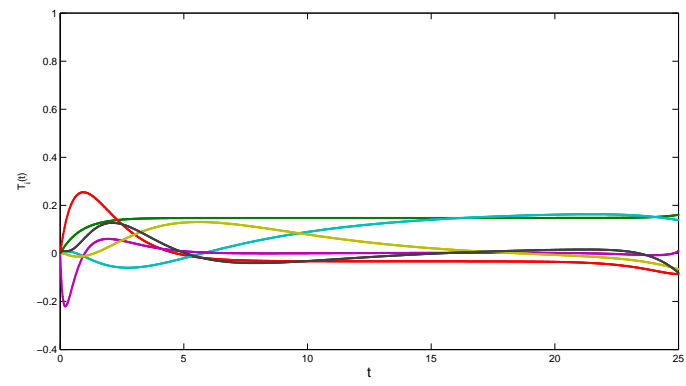

Figure 7: Seven most significant functions $T_{i}(t)$ involved in the Boltzmann solution separated representation.

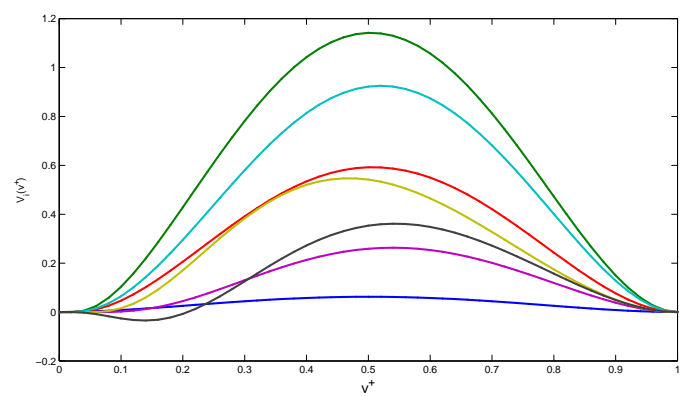

Figure 8: Seven most significant functions $V_{i}\left(v^{+}\right)$involved in the Boltzmann solution separated representation. 

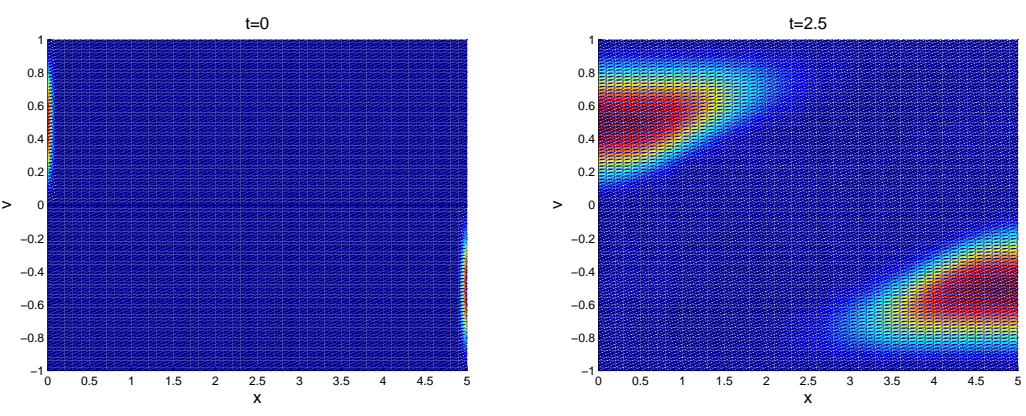

$t=5$
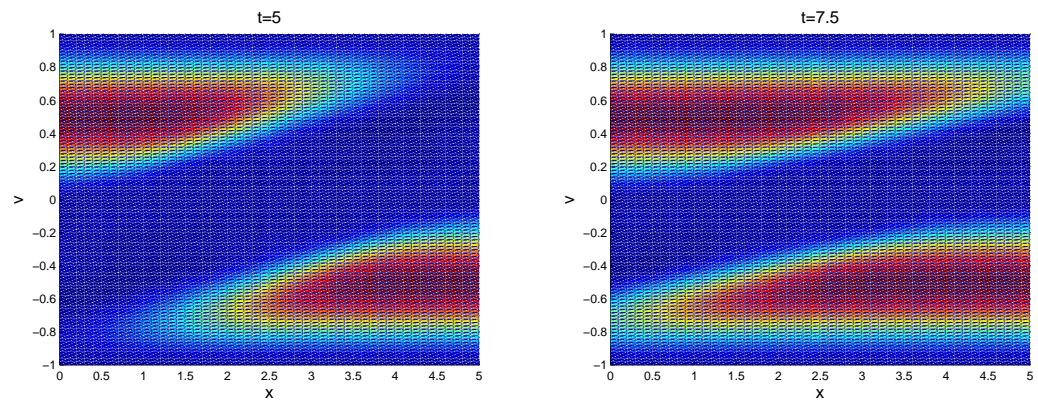

$t=10$

$t=20$
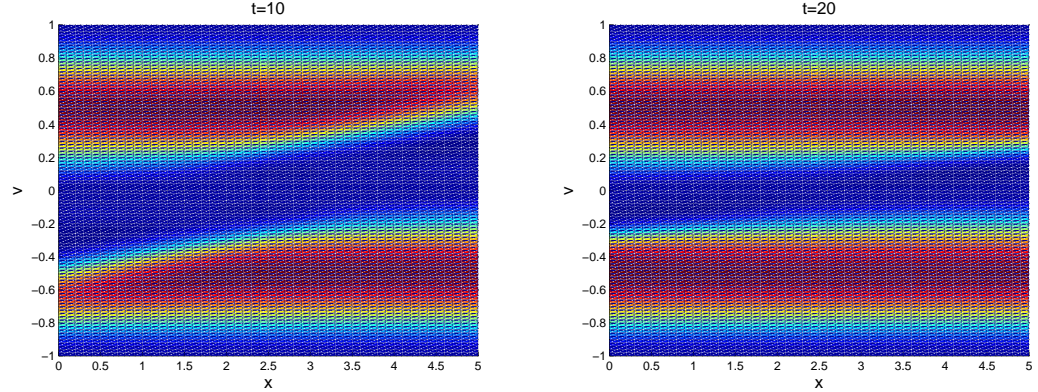

Figure 9: Reconstructed distributions $f(x, t, v)$ at times: (top-left) $t=0$, (top-right) $t=2.5$, (middle-left) $t=5$, (middle-right) $t=7.5$, (bottom-left) $t=10$ and (bottom-right) $t=20$.

\subsubsection{Convergence analysis}

In the context of separated representations the solution accuracy is determined by the space and time discretizations considered for describing the different functions involved in the decomposition as well as by the number of terms considered in the finite sum $N$. The effect of the mesh refinement was addressed in many of our former works. It was noticed that the converged separated representation approaches asymptotically the equivalent finite element solution that corresponds with a full tensor product of the approximation bases considered in each coordinate. Thus, the space (including the conformational space) and the time discretization must be defined in order to represent the main solution features (e.g. localization, boundary layers, etc.), and when the discretization cannot capture all the solution features appropriate adaptive strategies must be considered using adequate error estimators [8]. 


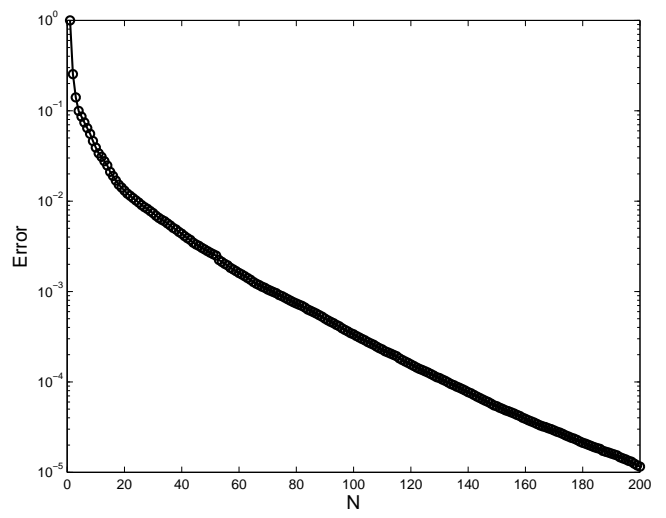

Figure 10: Convergence analysis: evolution of the equation residual (L2 norm) with the number of terms $N$ involved in the separated representation.

In what follows we are assuming a given mesh for the space, velocity and time and we are calculating the evolution of the partial differential equation residual (using a L2 norm) when increasing the number of terms involved in the finite sum decomposition $N$. In Fig. 10 it can be noticed that even in the transient case here addressed, the consideration of 20 terms reduces of 2 orders of magnitude the error (residual norm). It is important to note that this error concerns the whole space domain, the entire time interval and the whole velocity domain.

In that figure can be also noticed that adding much more terms has a limited impact in the accuracy enhancement because separated representation constructors reach a asymptotical behavior. As illustrated in [23] the best strategy for improving the solution consists in refining the discretization as soon as the asymptotical evolution is reached.

Even when the solution involves many terms separated representations proceed orders of magnitude faster than mesh-based discretization techniques as soon as the model dimension increases, as discussed in [23]. It is important to recall that in the present case we are solving a series of one-dimensional problems, that can be done extremely fast. Lattice-Boltzmann - LB - techniques are in fact excellent alternatives, but here we are precisely proposing techniques that could proceeds as fast as LB techniques while keeping a fine representation of the conformation space as discussed in the next section. Appropriate comparisons of the different available techniques constitutes a work in progress and it remains out of the scope of this work.

\subsection{D steady-state collision-free Boltzmann equation}

Finally we are considering the steady state collision-free Boltzmann problem in 2D

$$
\mathbf{v} \cdot \nabla_{x} f=0
$$

where $f(\mathbf{x}, \mathbf{v}), \mathbf{v}^{T}=(u, v)$ and $\mathbf{x}^{T}=(x, y)$. 


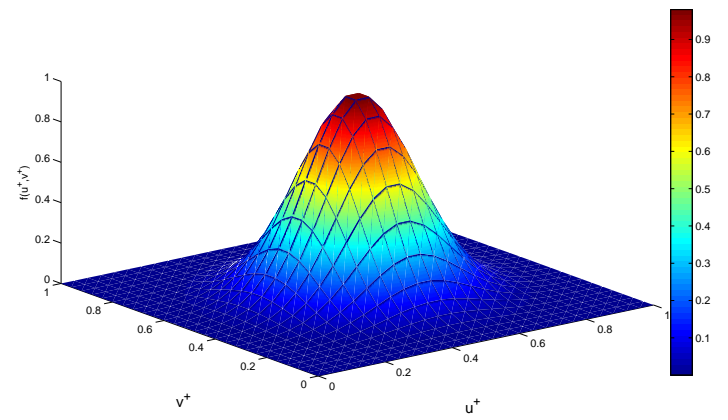

Figure 11: Velocity distribution enforced in the inflow boundaries of $\Omega_{x} \times \Omega_{v}$ in the 2D Boltzmann problem.

$(r, s)=(-1,+1)$

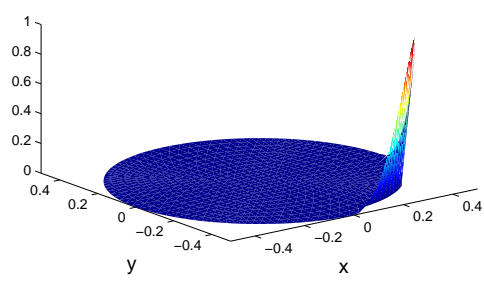

$(\mathrm{r}, \mathrm{s})=(-1,-1)$

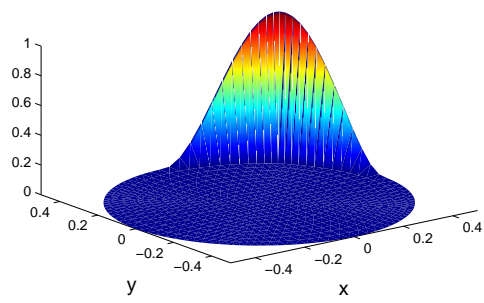

$(r, s)=(+1,+1)$

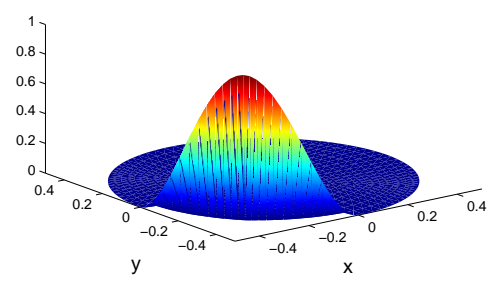

$(r, s)=(+1,-1)$

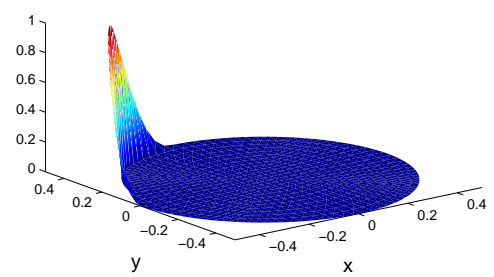

Figure 12: Function $X_{1}(x, y, r, s)$ for the 4 possible combinations of values of $r$ and $s$.

Following the same ideas we consider the separated representation of the distribution function $f(\mathbf{x}, \mathbf{v})$ according to:

$$
f(\mathbf{x}, \mathbf{v}) \approx \sum_{i=1}^{i=N} X_{i}(x, y, r, s) V_{i}\left(u^{+}, v^{+}\right),
$$

where $r$ and $s$ represent respectively the sign of $u$ and $v$, and consequently $r, s \in\{-1,1\}$.

The domain $\Omega_{x}$ is the circle of unit diameter centered at the origin of coordinates $\mathcal{O}=(0,0)$. The velocity domain is the square $\Omega_{v}=[-1,1] \times[-1,1]$. At the inflow boundary of $\Omega_{x} \times \Omega_{v}$ the velocity distribution depicted in Fig. 11 is enforced with the appropriate symmetries with respect to the axes $u=0$ and $v=0$ for defining it for the negative velocity components, multiplied by a given function of space (arbitrarily chosen). 


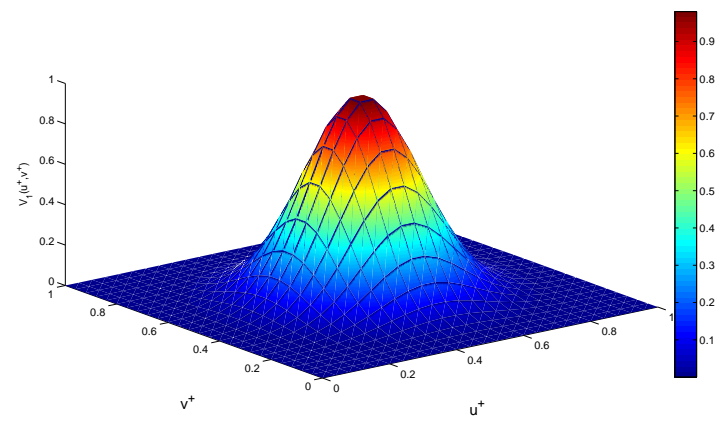

Figure 13: Function $V_{1}\left(u^{+}, v^{+}\right)$.

$(r, s)=(-1,+1)$

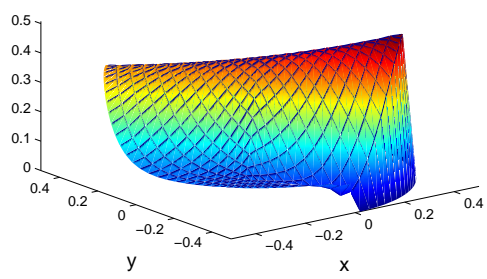

$(\mathrm{r}, \mathrm{s})=(-1,-1)$

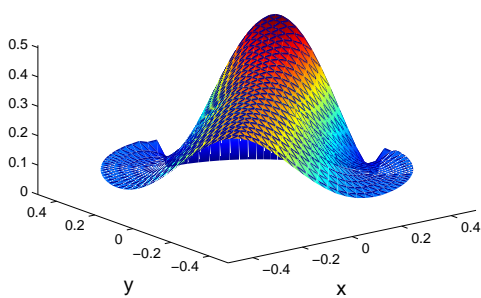

$(r, s)=(+1,+1)$

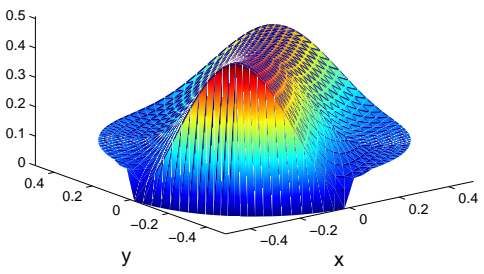

$(r, s)=(+1,-1)$

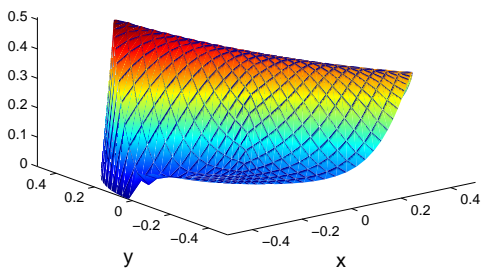

Figure 14: Function $X_{2}(x, y, r, s)$ for the 4 possible combinations of values of $r$ and $s$.

A first mode $X_{1}(x, y, r, s) V_{1}\left(u^{+}, v^{+}\right)$was defined in order to fulfill the Dirichlet boundary conditions, without taking care of its accuracy for representing the solution inside the domain $\Omega_{x} \times \Omega_{v}$. The functions involved in the first term of the finite sum (4.13), $X_{1}$ and $V_{1}$ are depicted in Figs. 12 and 13 respectively.

Now the enrichment procedure continues by calculating new terms of the separated representation until obtaining a solution accurate enough with respect to an appropriate error estimator (in the present case the residual $L^{2}$-norm). Fig. 14 shows the space function involved in the second term of the separated representation $X_{2}(x, y, r, s)$. Finally Fig. 15 depicts functions $V_{i}\left(u^{+}, v^{+}\right), i=2, \cdots, 5$.

Again, the solution of the $4 \mathrm{D}$ Boltzmann problem is obtained in few seconds in a laptop, thanks to the separated representation features. Its extension to 3D is straightforward as well as the introduction of appropriate collision terms. 

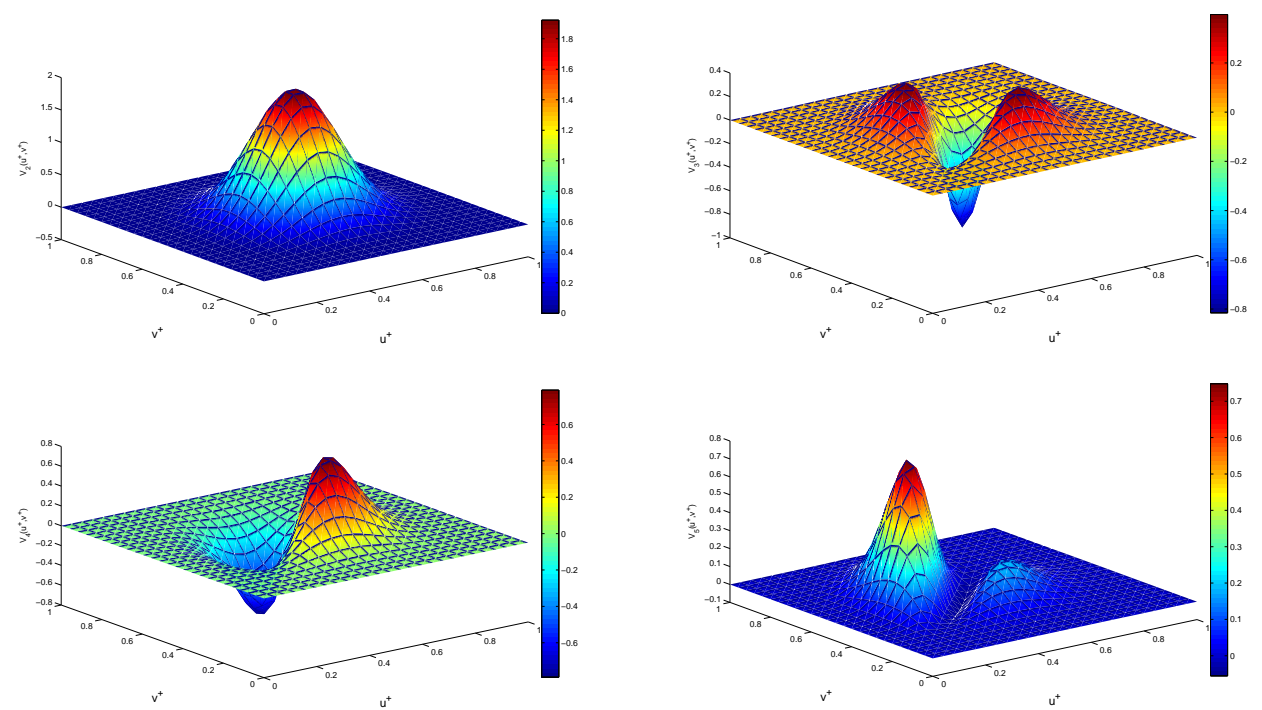

Figure 15: Functions $V_{i}\left(u^{+}, v^{+}\right)$: (top-left) $i=2$, (top-right) $i=3$, (bottom-left) $i=4$ and (bottom-right) $i=5$.

\subsection{Fully advective Fokker-Planck operator}

In this section we come back to the Fokker-Planck equation (3.1)

$$
\mathbf{v}(\mathbf{x}) \cdot \nabla \psi+\frac{\partial}{\partial \theta}(\dot{\theta} \psi)=0
$$

where we isolate the term involving the conformation coordinate $\theta$ in order to check the stabilized integration strategy described in Section 3. For this purpose we consider

$$
\frac{x}{|x|} \frac{\partial \psi}{\partial \theta}=|x| \cos \theta
$$

in $\Omega_{x} \times \Omega_{\theta}$, with $\Omega_{x}=[-1,0) \cup(0,1]$ and $\Omega_{\theta}=[0,2 \pi)$. The only condition applying in the $\theta$ direction concerns the solution periodicity.

The solution is searched in the separated form:

$$
\psi(x, \theta) \approx \sum_{i=1}^{N} X_{i}(x) \Theta_{i}(\theta)
$$

By using the strategy described in Section 3 the solution was obtained in one enrichment step, that is $N=1$ in Eq. (4.16), in agreement with the exact solution that reads $\psi^{e x}=x \sin \theta$. The solution $\psi(x, \theta)=X_{1}(x) \Theta_{1}(\theta)$ is depicted in Fig. 16 and both functions $X_{1}(x)$ and $\Theta_{1}(\theta)$ are depicted in Fig. 17. 


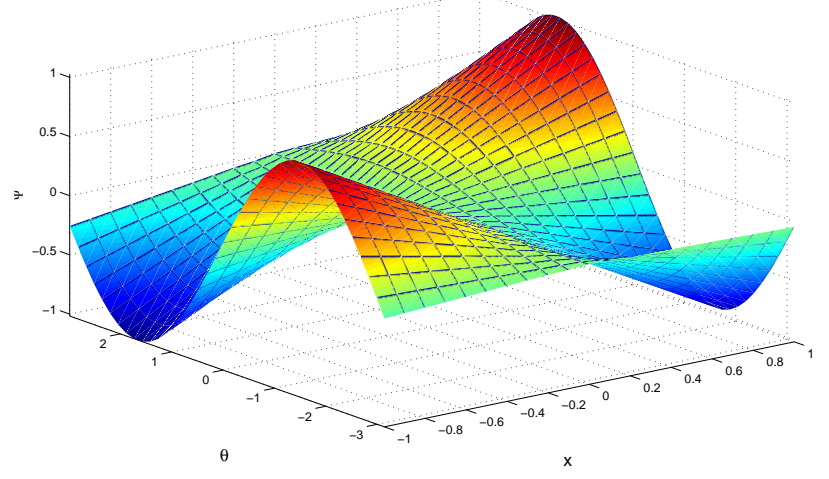

Figure 16: Solution $\Psi(x, \theta)=X_{1}(x) \Theta_{1}(\theta)$.
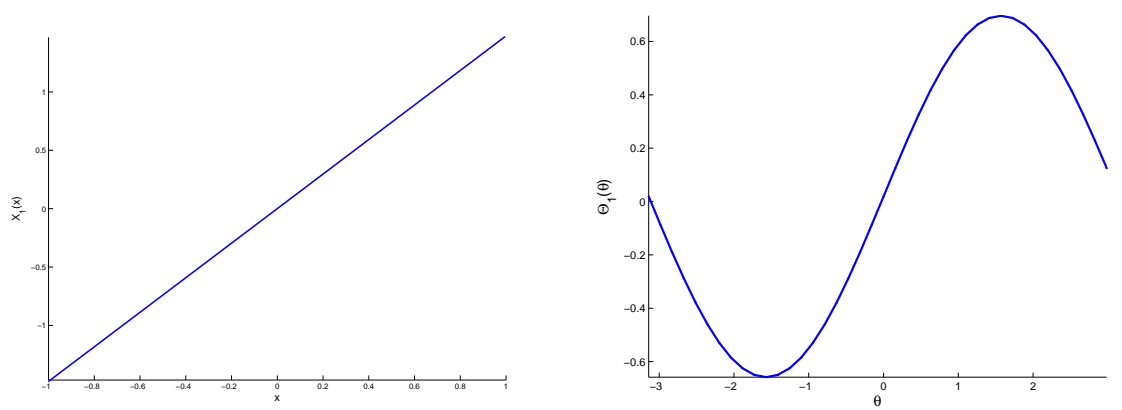

Figure 17: Functions $X_{1}(x)$ (left) and $\Theta_{1}(\theta)$ (right) involved in the separated representation of $\psi(x, \theta)$.

\section{Conclusions}

This paper addresses the challenging issue related to stabilization of discrete advective operators encountered in general Boltzmann and Fokker-Planck models. In the Boltzmann model the advection field is a conformation coordinate whereas in Fokker-Planck models the advection field consists of a field depending on the problem coordinates.

Thus the numerical treatment of both models is quite different. In this paper we proposed, described and tested efficient routes for addressing the just described issues. In the case of the Boltzmann models and inspired from the Lattice-Boltzmann practice we defined a conformation space including the velocity magnitude and its sign. Thus, the use of separated representation within the PGD framework allowed circumventing the curse of dimensionality within a stabilized discrete formulation.

In the case of the Fokker-Planck equation and prior to applied the PGD separated representation constructor we decomposed the advection field in order to guarantee stable discretizations within the PDG framework. 
Some numerical experiments allowed to conclude on the pertinence of the proposed approach.

\section{Acknowledgments}

Authors acknowledge the support of Spanish and French ministries through grants CICYTDPI2011-27778-C02-01/02 and ANR COSINUS 2010 SIMDREAM. F. Chinesta also acknowledge the Institut Universitaire de France - IUF.

\section{References}

[1] E. Abisset-Chavanne, R. Mezher, S. Le Corre, A. Ammar, F. Chinesta. Kinetic theory microstructure modeling in concentrated suspensions. Entropy, 15, 2805-2832, 2013.

[2] S. Aghighi, A. Ammar, C. Metivier, M. Normandin, F. Chinesta. Non-incremental transient solution of the Rayleigh-Bnard convection model using the PGD. Journal of Non-Newtonian Fluid Mechanics, 200, 65-78, 2013.

[3] A. Ammar, F. Chinesta, A particle strategy for solving Fokker-Planck equation governing the fiber orientation distribution in steady recirculating flows involving short fiber suspensions, Lecture Notes on Computational Science and Engineering. Springer, 43, 1-16, 2004.

[4] A. Ammar, B. Mokdad, F. Chinesta, R. Keunings. A new family of solvers for some classes of multidimensional partial differential equations encountered in kinetic theory modeling of complex fluids. Journal of Non-Newtonian Fluid Mechanics, 139, 153-176, 2006.

[5] A. Ammar, B. Mokdad, F. Chinesta, R. Keunings. A new family of solvers for some classes of multidimensional partial differential equations encountered in kinetic theory modeling of complex fluids. Part II: Transient simulation using space-time separated representation. Journal of Non-Newtonian Fluid Mechanics, 144, 98-121, 2007.

[6] A. Ammar, F. Chinesta, P. Joyot. The nanometric and micrometric scales of the structure and mechanics of materials revisited: An introduction to the challenges of fully deterministic numerical descriptions. International Journal for Multiscale Computational Engineering, 6/3, 191-213, 2008.

[7] A. Ammar, M. Normandin, F. Daim, D. Gonzalez, E. Cueto, F. Chinesta. Non-incremental strategies based on separated representations: Applications in computational rheology. Communications in Mathematical Sciences, 8/3, 671-695, 2010.

[8] A. Ammar, F. Chinesta, P. Diez, A. Huerta. An error estimator for separated representations of highly multidimensional models. Computer Methods in Applied Mechanics and Engineering, 199 1872-1880, 2010.

[9] A. Ammar, E. Cueto, F. Chinesta. Reduction of the Chemical Master Equation for gene regulatory networks using Proper Generalized Decompositions. International Journal for Numerical Methods in Biomedical Engineering, 28/9, 960-973, 2012.

[10] P.L. Bhatnagar, E.P. Gross, M. Krook. A model for collision processes in gases. I. Small amplitude processes in charged and neutral one-component systems. Phys. Rev., 94/3, 511-525, 1954.

[11] C.V. Chaubal, A. Srinivasan, O. Egecioglu, L.G. Leal. Smoothed particle hydrodynamics techniques for the solution of kinetic theory problems. J. Non-Newtonian Fluid Mech., 70, 125-154, 1997. 
[12] C. Chauviere, A. Lozinski. Simulation of dilute polymer solutions using a Fokker-Planck equation. Computers and Fluids., 33, 687-696. 2004.

[13] H.D. Chen, S.Y. Chen, W.H. Matthaeus. Recorvery of the Navier-Stokes equations using a Lattice-Gas Boltzmann Method. Physical Review A, 45/8, R5339-R5342, 1992.

[14] S. Chen, G.D. Doolen. Lattice Boltzmann method for fluid flows. Annual Review of Fluid Mechanics, 30, 329-364,1998.

[15] F. Chinesta, G. Chaidron, A. Poitou. On the solution of the Fokker-Planck equations in steady recirculating flows involving short fiber suspensions. J. Non-Newtonian Fluid Mech., 113/23, 97-125, 2004.

[16] F. Chinesta, A. Ammar, A. Falco, M. Laso. On the reduction of stochastic kinetic theory models of complex fluids. Modeling and Simulation in Materials Science and Engineering, 15, 639-652, 2007.

[17] F. Chinesta, M. Mackley. Microstructure evolution during liquid-liquid laminar mixing: A kinetic theory approach. International Journal of Material Forming, 1, 47-55, 2008.

[18] F. Chinesta, A. Ammar, E. Cueto. Recent advances and new challenges in the use of the Proper Generalized Decomposition for solving multidimensional models. Archives of Computational Methods in Engineering, 17/4, 327-350, 2010.

[19] F. Chinesta, A. Ammar, A. Leygue, R. Keunings. An overview of the Proper Generalized Decomposition with applications in computational rheology. Journal of Non Newtonian Fluid Mechanics, 166, 578-592, 2011.

[20] F. Chinesta, P. Ladeveze, E. Cueto. A short review in model order reduction based on Proper Generalized Decomposition. Archives of Computational Methods in Engineering, 18, 395404, 2011.

[21] F. Chinesta, A. Leygue, F. Bordeu, J.V. Aguado, E. Cueto, D. Gonzalez, I. Alfaro, A. Ammar, A. Huerta. PGD-based computational vademecum for efficient design, optimization and control. Arch. Comput. Methods Eng., 20, 31-59, 2013

[22] F. Chinesta. From single-scale to two-scales kinetic theory descriptions of rods suspensions. Archives in Computational Methods in Engineering, 20/1, 1-29, 2013.

[23] F. Chinesta, R. Keunings, A. Leygue. The Proper Generalized Decomposition for advanced numerical simulations. A primer. Springerbriefs, Springer, 2014.

[24] J.J. Derksen, Prashant. Simulations of complex flow of thixotropic liquids. Journal of Non Newtonian Fluid Mechanics, 160/2-3, 65-75, 2009.

[25] D. Dhumieres, P. Lallemand. Lattice Gas Automata For Fluid-Mechanics. Physica A, 140/12, 326-335, 1986.

[26] P. Espanol. Statistical Mechanics of Coarse-Graining. In Novel Methods in Soft Matter Simulations. M. Karttunen, A. Lukkarinen, and I. Vattulainen, Eds. Lecture Notes in Physics, vol 640, pages 69-115. Springer Berlin Heidelberg, 2004.

[27] M. Grmela, F. Chinesta, A. Ammar. Mesoscopic tube model of fluids composed of wormlike micelles. Rheologica Acta, 49/5, 495-506, 2010.

[28] M. Grmela, A. Ammar, F. Chinesta. One and two-fiber orientation kinetic theories of fiber suspensions. Journal of Non-Newtonian Fluid Mechanics, 200, 17-33, 2013.

[29] X.Y. He, L.S. Luo, M. Dembo. Some progress in lattice Boltzmann method. 1. Nonuniform mesh grids. Journal of Computational Physics, 129/2, 357-363, 1996.

[30] X.Y. He, L.S. Luo. Theory of the lattice Boltzmann method: From the Boltzmann Equation to the lattice Boltzmann equation. Physical Review E, 56/6, 6811-6817, 1997.

[31] F. J. Higuera and J. Jimnez. Boltzmann Approach to Lattice Gas Simulations. Europhys. Lett. 9 663, 1989. doi:10.1209/0295-5075/9/7/009 
[32] S.L. Hou, Q. Zou, S.Y. Chen, G. Doolen, A.C. Cogley. Simulation of cavity flow by the Lattice Boltzmann Method. Journal of Computational Physics, 118/2, 329-347, 1995.

[33] T. Inamuro, T. Ogata, S. Tajima, N. Konishi. A lattice Boltzmann method for incompressible two-phase flows with large density differences. Journal of Computational Physics,198/2, 628-644, 2004.

[34] R. Jendrejack, J. de Pablo, M. Graham. A method for multiscale simulation of flowing complex fluids. J. Non-Newtonian Fluid Mech., 108, 123-142, 2002.

[35] R. Keunings, Micro-macro methods for the multiscale simulation viscoelastic flow using molecular models of kinetic theory, Rheology Reviews, D.M. Binding and K. Walters (Edts.), British Society of Rheology, 67-98, 2004.

[36] A.J.C. Ladd, R. Verberg. Lattice-Boltzmann simulations of particle-fluid suspensions. Journal of Statistical Physics, 104/5-6, 1191-1251, 2001.

[37] P. Lallemand, L.S. Luo. Theory of the lattice Boltzmann method: Dispersion, dissipation, isotropy, Galilean invariance, and stability. Physical Review E, 61/6, 6546-6562, 2000.

[38] H. Lamari, A. Ammar, A. Leygue, F. Chinesta. On the solution of the multidimensional Langer's equation by using the Proper Generalized Decomposition Method for modeling phase transitions. Modelling and Simulation in Materials Science and Engineering, 20, 015007, 2012.

[39] A. Lozinski, C. Chauviere. A fast solver for Fokker-Planck equation applied to viscoelastic flows calculations: 2D FENE model. J. Computational Physics, 189, 607-625, 2003.

[40] G.R. McNamara, G. Zanetti. Use of the Boltzmann-Equation to Simulate Lattice-Gas Automata. Physical Review Letters, 61/20, 2332-2335, 1988.

[41] G. Maitejean, A. Ammar, F Chinesta, M. Grmela. Deterministic solution of the kinetic theory model of colloidal suspensions of structureless particles. Rheologica Acta, 51/6, 527-543, 2012.

[42] G. Maitrejean, A. Ammar, F. Chinesta. Simulating microstructural evolution during pasive mixing. Internantional Journal of Material Forming, 5/1, 73-81, 2012.

[43] G. Maitrejean, M. Grmela, A. Ammar, F. Chinesta. Kinetic theory of colloidal suspensions: Morphology, rheology and migration. Rheologica Acta, 52/6, 557-577, 2013.

[44] N.S. Martys, H.D. Chen. Simulation of multicomponent fluids in complex three-dimensional geometries by the lattice Boltzmann method. Physical Review E, 53/1, 743-750, 1996.

[45] B. Mokdad, E. Pruliere, A. Ammar, F. Chinesta. On the simulation of kinetic theory models of complex fluids using the Fokker-Planck approach. Applied Rheology,17/2, 26494, 1-14, 2007.

[46] B. Mokdad, A. Ammar, M. Normandin, F. Chinesta, J.R. Clermont. A fully deterministic micro-macro simulation of complex flows involving reversible network fluid models. Mathematics and Computer in Simulation, 80, 1936-1961, 2010.

[47] R.R. Nourgaliev, T.N. Dinh, T.G. Theofanous, D. Joseph. The lattice Boltzmann equation method: theoretical interpretation, numerics and implications. International Journal of Multiphase Flow, 29/1, 117-169, 2003.

[48] H. C. Öttinger, M. Laso. Smart polymers in finite element calculation, Int Congr. on Rheology, Brussel, Belguim, 1992.

[49] E. Pruliere, A. Ammar, N. El Kissi, F. Chinesta. Recirculating flows involving short fiber suspensions: Numerical difficulties and efficient advanced micro-macro solvers. Archives of Computational Methods in Engineering, State of the Art Reviews, 16, 1-30, 2009.

[50] M. Rohde, J.J. Derksen, H.E.A. Van den Akker. Volumetric method for calculating the flow around moving objects in Lattice-Boltzmann schemes. Physical Review E, 65/5, 2002. 
[51] M. Rohde, D. Kandhai, J.J. Derksen, H.E.A. van den Akker. A generic, mass conservative local grid refinement technique for Lattice-Boltzmann schemes. International Journal for Numerical Methods in Fluids, 51/4, 439-468, 2006.

[52] M. Somasi, B. Khomami, N.J. Woo, J.S. Hur, E.S.G. Shaqfeh. Brownian dynamics simulations of bead-rod and bead-spring chains: numerical algorithms and coarse-graining issues. J. Non-Newtonian Fluid Mech., 108/1-3, 227-255, 2002.

[53] S. Succi.The Lattice Boltzmann Equation For Fluid Dynamics and Beyond. Clarendon Press, 2001

[54] G. Venkiteswaran, M. Junk, A QMC approach for high dimensional Fokker-Planck equations modelling polymeric liquids. Mathematics and Computers in Simulation, 68, 43-56, 2005.

[55] R. Verberg, A.J.C. Ladd. Simulation of low-Reynolds-number flow via a time-independent Lattice-Boltzmann method. Physical Review E, 60/3, 3366-3373, 1999.

[56] D.Z. Yu, R.W. Mei, L.S. Luo, W. Shyy. Viscous flow computations with the method of lattice Boltzmann equation. Progress in Aerospace Sciences, 39/5, 329-367, 2003. 